\title{
Sentinel-1 Additive Noise Removal from Cross-Polarization Extra-Wide TOPSAR with Dynamic Least-Squares
}

\author{
Peter Q. Lee ${ }^{\mathrm{a}}$, Linlin $\mathrm{Xu}^{\mathrm{a}}$, David A. Clausi ${ }^{\mathrm{a}}$ \\ ${ }^{a}$ Department of Systems Design Engineering, Faculty of Engineering, University of Waterloo, 200 University Avenue West Ontario, \\ Canada, N2L $3 G 1$
}

\begin{abstract}
Sentinel-1 is a synthetic aperture radar (SAR) platform with an operational mode called extra wide (EW) that offers large regions of ocean areas to be observed. A major issue with EW images is that the cross-polarized HV and VH channels have prominent additive noise patterns relative to low backscatter intensity, which disrupts tasks that require manual or automated interpretation. The European Space Agency (ESA) provides a method for removing the additive noise pattern by means of lookup tables, but applying them directly produces unsatisfactory results because characteristics of the noise still remain. Furthermore, evidence suggests that the magnitude of the additive noise dynamically depends on factors that are not considered by the ESA estimated noise field.

To address these issues we propose a quadratic objective function to model the mis-scale of the provided noise field on an image. We consider a linear denoising model that re-scales the noise field for each subswath, whose parameters are found from a least-squares solution over the objective function. This method greatly reduces the presence of additive noise while not requiring a set of training images, is robust to heterogeneity in images, dynamically estimates parameters for each image, and finds parameters using a closed-form solution.

Two experiments were performed to validate the proposed method. The first experiment simulated noise removal on a set of RADARSAT-2 images with noise fields artificially imposed on them. The second experiment conducted noise removal on a set of Sentinel-1 images taken over the five oceans. Afterwards, quality of the noise removal was evaluated based on the appearance of open-water. The two experiments indicate that the proposed method marks an improvement both visually and through numerical measures. 11
\end{abstract}

Keywords: Sentinel-1, SAR, Noise removal, Denoising, Additive noise, Thermal noise, Banding, Scalloping

\section{Introduction}

Synthetic aperture radar (SAR) is method of remote sensing that is useful for monitoring the surface of the planet while being insensitive to atmospheric conditions. Applications include topographical mapping through SAR interferometry (Bailey, 2014: Pepe and Calò, 2017), sea ice mapping/classification (Wang et al., 2017; Tan et al. 2018: Hong and Yang, 2018, $\mathrm{Xu}$ and Li, 2015, Yu and Clausi, 2008), oil spill detection (Xu et al., 2016; Topouzelis, 2008), ship detection (Li et al., 2018, Pelich et al., 2015), and others (Kasischke et al., 1997; Elachi et al., 1982). Consequently, SAR imagery is an essential tool for global monitoring. The Sentinel-1 program, which was created and is administered by the European Space Agency (ESA), operates two SAR satellites, Sentinel-1A (launched 2014) and Sentinel-1B (launched 2016), and acquires and distributes SAR images taken over Earth. The diverse set of applications for SAR and the fact that the Sentinel program provides open-data solidifies the potential for research and commercial products.

Sentinel-1 operates in a number of acquisition modes. This paper focuses on the Sentinel-1 extra-wide (EW) mode, which is mainly used over ocean regions, with the images acquired using a method called TOPSAR (Zan and Guarnieri, 2006). Sentinel-1 satellites emit bursts of horizontally or vertically polarized radiation and receives polarized backscatter to form co-polarized images, where the polarization of the emission is the same as the polarization of the received signal, or cross-polarized images, where the polarization of the emission is orthogonal to the polarization of the received signal. A caveat of EW mode for Sentinel-1 is that there are prominent additive noise patterns present in the cross polarization channels with respect to lower backscatter intensity (Piantanida,

${ }^{1}$ This manuscript has been accepted in Remote Sensing of Environment, whose version of record can be found at https://doi. org/10.1016/j.rse.2020.111982 (C)2020. This manuscript version is made available under the CC-BY-NC-ND 4.0 license http: //creativecommons.org/licenses/by-nc-nd/4.0/ 
2017b; Park et al., 2018, Tan et al., 2018). These are sometimes called banding or scalloping effects in the literature (Wang et al. 2017: Wei and Chen, 2007). These noise patterns can cause major issues for interpretation-based tasks because the added intensity corrupts the true backscattered signal of the target (Piantanida, 2017b). ESA currently provides lookup tables to estimate the additive noise in terms of azimuth and range components as detailed in their Instrument Processing Facility (IPF) product (Piantanida, 2017b), and disseminated through noise calibration $e X$ tensible Markup Language (XML) files that are distributed with each Sentinel-1 scene. By interpolating the lookup tables, an estimated additive noise field can be derived for the original image. This paper focuses specifically on data using IPF version 2.91, however as of writing IPF version 3.20 is the latest version that presents improvements in computing the additive noise field. Working with data from IPF versions less than or equal to 2.91 is problematic, however, as subtracting the noise field from the image directly is insufficient as shown by others (Park et al., 2018, Tan et al. 2018, Karvonen, 2017) and ourselves in Section 3. The problem is severe enough that some practitioners prefer to discard the first subswath to make modeling easier (Tan et al. 2018, Hong and Yang, 2018).

While there is a significant body of work aiming to reduce speckle noise (Gagnon and Jouan, 1997; Parrilli et al. 2011; Qiu et al., 2004, Agrawal and Venugopalan, 2011; Santoso et al., 2015; Marhaba and Zribi, 2018; Maity et al. 2015), there are considerably fewer published methods on reducing the additive noise in SAR images.

Iqbal et al. (2012) modeled the additive noise field of ScanSAR images with gain and offset parameters and implemented a Kalman filter to estimate the parameters for the azimuth and range in a decoupled manner. However, it is not immediately clear how this could be extended to Sentinel-1 EW where the magnitude of the additive noise varies greatly between adjacent subswaths. Specific to Sentinel-1 EW, Karvonen (2017) used an approach with a finite impulse response filter to reduce the scalloping in the azimuth direction selectively over ocean regions. However, this has the computationally expensive requirement of computing texture features, with the possibility of incorrectly modifying the intensity of the intended target within the image. Park et al. (2018) created an approach for Sentinel-1 EW that modifies the estimated noise field with linear scaling and intercept parameters in an attempt to eliminate the additive noise in the image. The values of the parameters were estimated with an iterative grid search regression approach using a large sample of Sentinel-1 ocean scenes, with the mean-estimates of the parameters used for evaluation. A few drawbacks of this approach include the requirement of selecting samples of images across uniform ocean areas, a priori. The method also makes the assumption that the ideal scaling parameters are the same for every scene, an assumption that we found to not always be valid, with an example of this shown in Fig. 4. Finally, if one wanted to adapt the method to dynamically estimate the parameters for individual scenes, it would be limited because of the aforementioned uniform ocean requirement.

The method proposed in this paper builds upon the approach of scaling the noise field for each subswath as proposed by Park et al. (2018). Our contribution is based both on creating an objective function that incorporates the characteristics of the additive noise and simultaneously choosing scaling parameters for each subswath to minimize the objective function. Our method leads to four main aspects of improvement:

1. Parameters may be estimated on images with heterogeneous features (e.g. containing open-water and sea-ice, cyclones, etc.).

2. No training set of images is needed; the method can be applied for each individual image without prior knowledge of the scenes.

3. Parameters are dynamically estimated for each image. This is significant because the ideal scaling is different for each image, which makes using static parameter estimates suboptimal.

4. The method has a closed-form solution that can be solved exactly without iterative methods.

Ultimately our approach reduces to estimating the parameters of a linear function based on a quadratic objective function using a least-squares solution.

Two experiments were performed to evaluate the effectiveness of the proposed denoising method. The first experiment simulated denoising by applying randomly scaled noise fields to non-SAR Sentinel-2 images and then denoising them with the proposed method. The second experiment used a sample of HV and VH polarized Sentinel-1 EW images from all five oceans and evaluated the proposed method in terms of the flatness of the result over selected subregions that were expected to be flat. These experiments ultimately indicated that the proposed method was a significant improvement over the baseline method provided by ESA and also that estimating parameters for each image independently is beneficial compared to a static estimation approach. We also perform a small experiment on data processed with an IPF above 3 that resulted in some modest improvement. Therefore, this work is useful for any application that uses EW cross-polarized Sentinel-1, such as ice analysis, as the method provides significantly better denoising and does not require expensive computation or large amounts of prior information or training sets. 


\section{SAR noise background}

Before details on the mechanics of the noise are provided, a brief background of TOPSAR will first be given. TOPSAR is a type of SAR acquisition that captures a wide swath area without the typical loss of resolution or scalloping (Zan and Guarnieri, 2006). The method works by simultaneously acquiring subswaths that cover different elevation angles (the angle between the ground target and the satellite) by steering the antenna aperture to focus at different elevation angles while continuously rotating along the azimuth angle. Sentinel-1 incorporates TOPSAR in two modes, interferometric wide (IW) swath mode, and extra wide (EW) swath mode. EW mode has subswaths enumerated as EW1, EW2, EW3, EW4, and EW5 that are ordered by increasing elevation angle along the range. During the observation period, the satellite sweeps a number of radar bursts along the azimuth and observes the backscattered radiation for each of the five subswaths. The received signals from each of the bursts are stitched along the azimuth direction (the direction of the satellite's orbital velocity) to form the rows of the image, while the signal corresponding to the five subswaths along the range (the direction perpendicular to the satellite's orbital velocity and tangent to the Earth's surface) are stitched together to form the columns of the image. In the supplementary materials we provide an animation that approximates the TOPSAR process.

ESA provides a forward model for estimating the additive noise induced by TOPSAR as a function of a number of factors

$$
y_{a}(i, j)=\frac{D(i) P(a, i)}{E(a, i, j) R(j)} f,
$$

for azimuth index $i$, range index $j$ that are within a subswath $a$ (Piantanida, 2017a). $P(a, i)$ is the power gain term that is used for drift correction while orbiting. $R(j)$ is the range spreading loss term used to correct errors from the range compression algorithm. Scalar $f$ is calibration parameter determined by the noise and the processor. The remaining two terms are based on the radiation pattern of the antennas, with $D(i)$, the descalloping gain, being inversely proportional to the antenna array pattern with respect to the azimuth, and $E(a, i, j)$, the elevation antenna pattern gain, being proportional to the antenna pattern with respect to the range. The reader is referred to Piantanida (2017a) for additional details. In antenna theory, the power of the radiation pattern for an antenna is directly related to the strength of the received signal at the corresponding angle from the receiving antenna (Visser, 2012). Consequently, the radiation pattern is a fundamental variable for the shape of the additive noise.

As Sentinel-1 uses an array antenna, the observed signal in the azimuth direction will naturally be an aggregate function of the radiation patterns for each element in the array as they are rotated along the azimuth (Piantanida, 2017a Visser, 2012). Fig. 1a shows an example of the relationship between the average antenna element pattern for the burst along the azimuth, derived from the Sentinel-1 calibration XML file, and azimuth component of the estimated noise. As the bursts are stitched along the azimuth (Zan and Guarnieri, 2006), the aggregate pattern appears as U-shaped and symmetric. The corresponding angles closer to the burst centre have higher gain, which reduces the magnitude of additive noise compensation needed. Angles further from the centre have less antenna-gain and require more noise compensation.

The negative relationship between the noise and radiation pattern is apparent in the range direction as well. Fig. $1 \mathrm{~b}$ shows a comparison between the range noise and the radiation pattern for each subswath. When the antenna is steered to acquire a different elevation angle, and hence different subswath, the subsequent radiation pattern changes. This is most notable in the first subswath, where the radiation pattern has two main lobes (local maxima of the gain). The remaining subswaths only have one main lobe, but have different relative magnitudes of radiation power. 

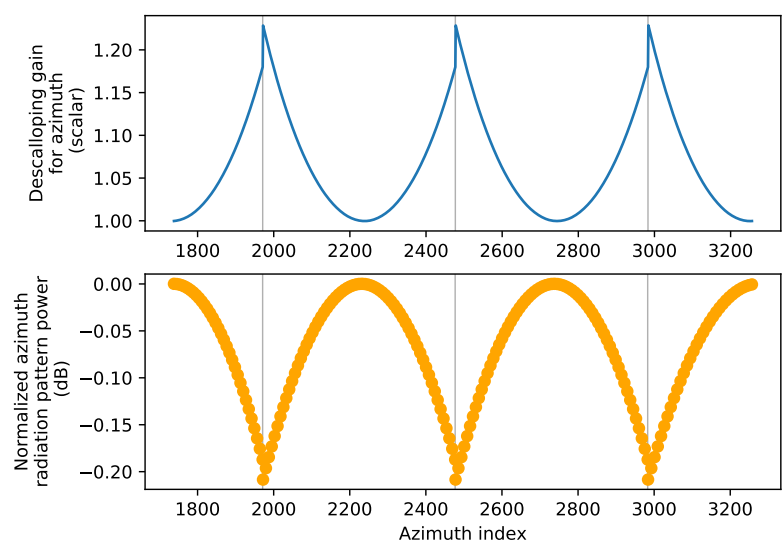

(a) Top: The estimated noise contribution in the azimuth direction (descalloping gain).

Bottom: Average radiation pattern power gain with respect to azimuth.

The vertical demarcations indicate the beginning and end of observations obtained from a burst. Only a portion of the azimuth is shown for brevity.


(b) Top: The estimated noise contribution in the range direction. Bottom: Radiation pattern power gain with respect to range. The vertical demarcations indicate the beginning and end of subswaths.

Figure 1: Comparison between the estimated noise contributed by the azimuth and range with respect to radiation pattern for EW TOPSAR. The measurements for the antenna pattern were initially given in angles and were converted to the corresponding range and azimuth indices in order to show the negative relationship between noise and radiation pattern. The measurements correspond to the noise field as shown in Fig. 2

Within the noise calibration XML files in every Sentinel-1 EW product, as of IPF 2.9, the information to compute the noise field $y_{a}(i, j)$ is provided within two different lookup tables labelled as the noiseRangeVector, which models $y_{a}(i, j) / D(i)$, and the noiseAzimuthVector, which models $D(i)$. By performing linear interpolation between entries in the lookup tables and multiplying the two results together, the estimated noise field $y_{a}(i, j)$ can be constructed. Given $x$ as the square of the digital pixel values in the original image, ESA recommends denoising the measurements by subtracting the noise field from the image

$$
\phi_{a}(i, j)=x_{a}(i, j)-y_{a}(i, j),
$$

where the square root of $\phi$ returns the result to the original linear units / digital values (Piantanida, 2017b). As this step is performed prior to any other calibration procedures, which differ based on application, we will continue to operate in terms of linear units throughout this paper. Further, for a given subswath $a \in \mathscr{A}=$ $\{\mathrm{EW} 1, \mathrm{EW} 2, \mathrm{EW} 3, \mathrm{EW} 4, \mathrm{EW} 5\}$, let the azimuth row and range column be $(i, j) \in a$. Then 2 is represented more succinctly as

$$
\phi_{a}=x_{a}-y_{a} .
$$

However, applying (3) directly is insufficient. Fig. 2 provides a visualization of the process of constructing the noise field and the result of subtracting it from the original image. The subtraction results in subswaths being under or over compensated. For example, Fig. 3 shows the values along the azimuth and range directions of an open-water area, where the result has noise patterns still present. This implies that the strength of the noise field needs to be scaled differently for each subswath. Park et al. (2018) proposed a method that chooses vectors for scaling coefficients $\overline{\mathbf{k}}=\left[\bar{k}_{\mathrm{EW} 1}, \bar{k}_{\mathrm{EW} 2}, \bar{k}_{\mathrm{EW} 3}, \bar{k}_{\mathrm{EW} 4}, \bar{k}_{\mathrm{EW} 5}\right]$ and intercepts $\overline{\mathbf{o}}=\left[\bar{o}_{\mathrm{EW} 1}, \bar{o}_{\mathrm{EW} 2}, \bar{o}_{\mathrm{EW} 3}, \bar{o}_{\mathrm{EW} 4}, \bar{o}_{\mathrm{EW} 5}\right]$ such that

$$
\bar{\phi}_{a}=x_{a}-\bar{k}_{a} y_{a}+\bar{o}_{a}
$$

where $\overline{\mathbf{k}}$ and $\overline{\mathbf{o}}$ were statically estimated using a training set of several hundred images. 


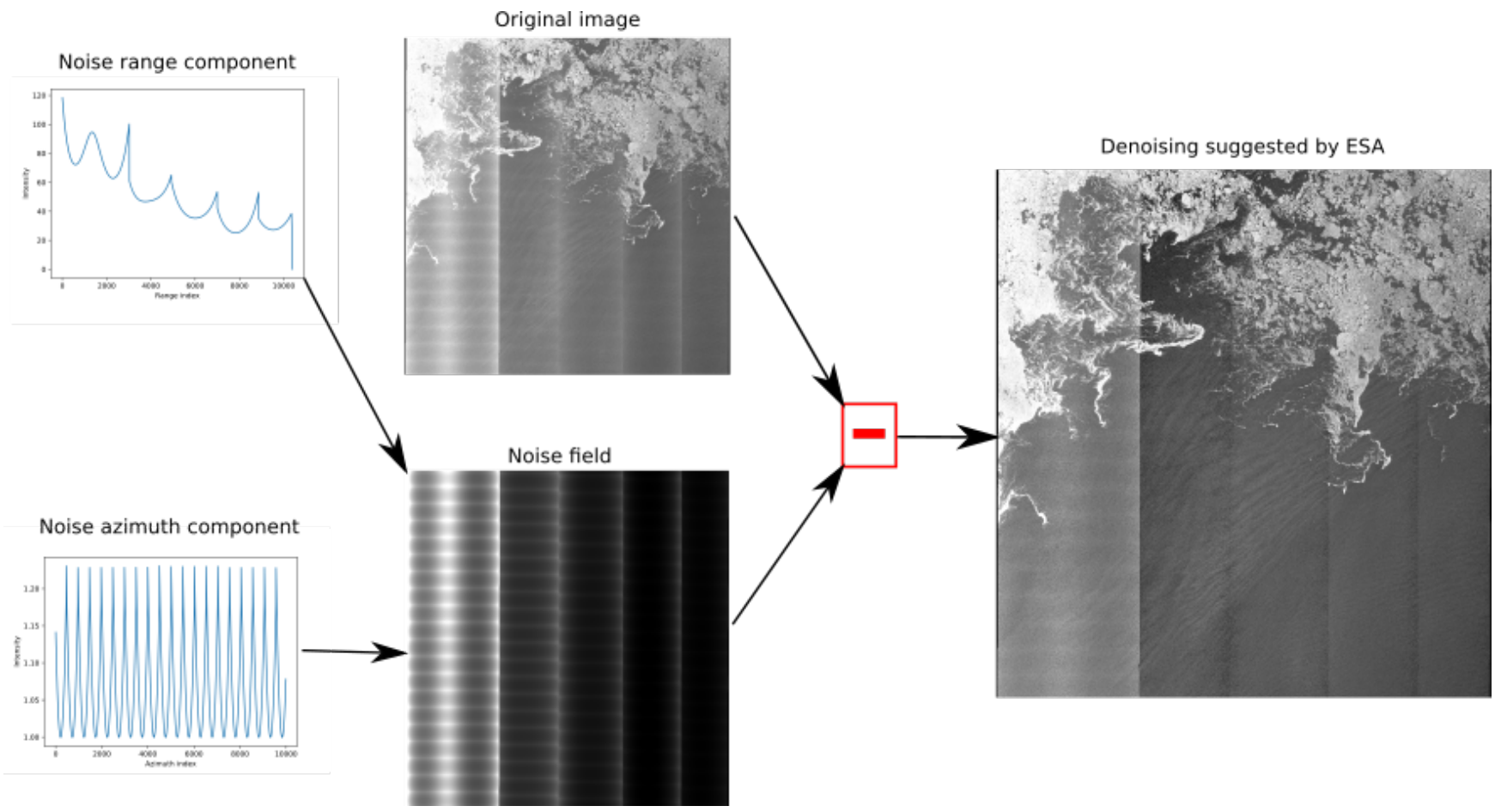

Figure 2: Visualization of the construction and application of the noise field using the noise vectors. Overall, the final denoising is unsatisfactory, most prominently in EW1 where patterns of the noise field are blatant in the result image.

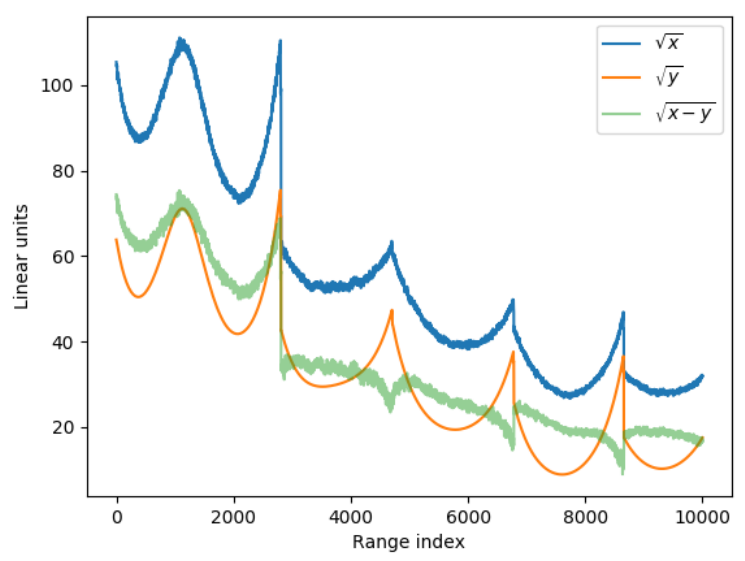

(a) Mean intensity of measurement, noise, and their difference over ocean area with respect to Range.

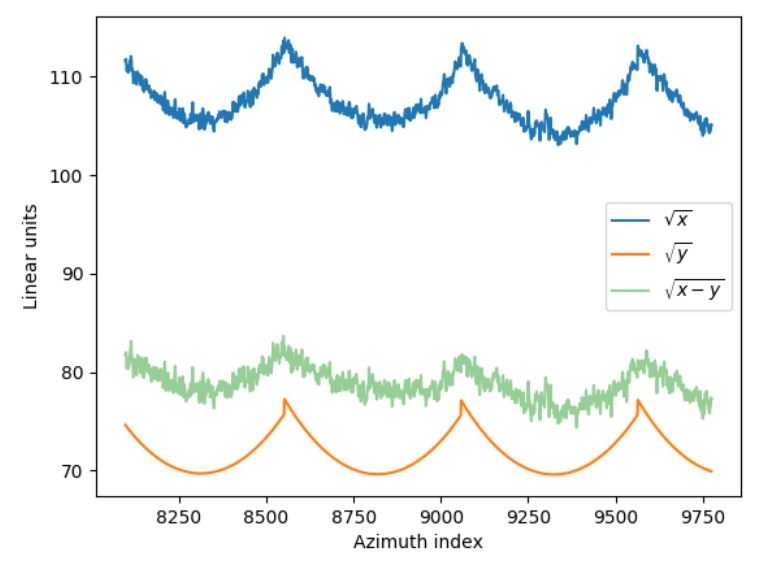

(b) Mean intensity of measurement, noise, and their difference over ocean area with respect to Azimuth for EW1.

Figure 3: Plots of mean intensities of measurements in sections of ocean with respect to each direction. Ideally the subtraction would produce a flat profile, but aspects of the noise are still present in both directions.

Another element to consider is whether the ideal scaling parameters are dependent on factors other than the sensor. Fig. 4 shows that single values of $k$ do not universally fit between different images. Therefore, it would be ideal for scaling factors to be estimated dynamically for each image. 


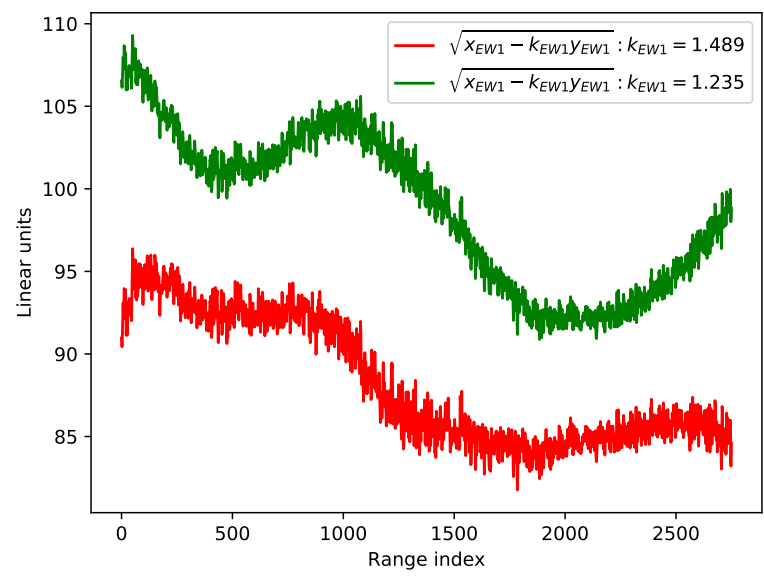

(a) Scene with a higher magnitude of additive noise.

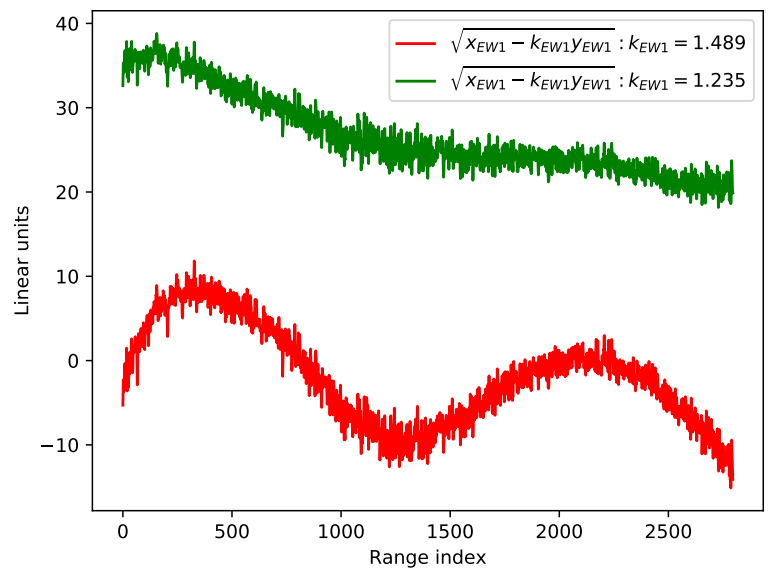

(b) Scene with a lower magnitude of additive noise.

Figure 4: A comparison of the mean measurement of EW1 over ocean regions of two different scenes after noise removal with two different scaling factors $(k)$. At $k=1.2350$ the left scene has noise patterns present while the right scene has a flat profile. However, at $k=1.4886$ the left scene as the noise patterns better compensated, but results in the right scene being overcompensated. This indicates that no single scaling factor will fit every scene.

\section{Methods}

The goal of this work is to estimate appropriate scaling parameters for each image individually. We make a similar assumption to Park et al. (2018) that the estimated noise in each subswath needs to be linearly re-scaled. Our dynamic model uses a set of dynamically estimated scaling parameters $\hat{\mathbf{k}}=\left[\hat{k}_{\mathrm{EW} 1}, \hat{k}_{\mathrm{EW} 2}, \hat{k}_{\mathrm{EW} 3}, \hat{k}_{\mathrm{EW} 4}, \hat{k}_{\mathrm{EW} 5}\right]$ such that

$$
\hat{\phi}_{a}=x_{a}-\hat{k}_{a} y_{a}
$$

Our model is distinct from (4) because the scaling parameters, $\hat{\mathbf{k}}$, are estimated for each image independently. We also considered using a set of intercepts ô but found that including these did not make a significant impact on the end results. To estimate parameters for each image independently, an objective function, $L$, is defined based on the characteristics of the estimated additive noise present within each image. The values of $\hat{\mathbf{k}}$ are thereby chosen to minimize $L$ such that $\hat{\mathbf{k}}=\operatorname{argmin} L$.

$\hat{\mathbf{k}}$

Throughout the remainder of this section we describe the main components of the objective function (L) based on the noise characteristics in both the azimuth and range directions. Thus, $L$ is defined as the sum of terms based on the noise-characteristics in the azimuth direction $\left(L^{A}\right)$, the range direction $\left(L^{R}\right.$ and $L^{B}$ that correspond to effects within and between subswaths respectively), and a term for regularization $\left(L^{r}\right)$. Each of these terms involve summing the square difference between different pairs of samples within the images. These terms are defined with more detail in the remainder of this section, but Fig. 5 provides a high-level visual overview of how $L$ is computed. 


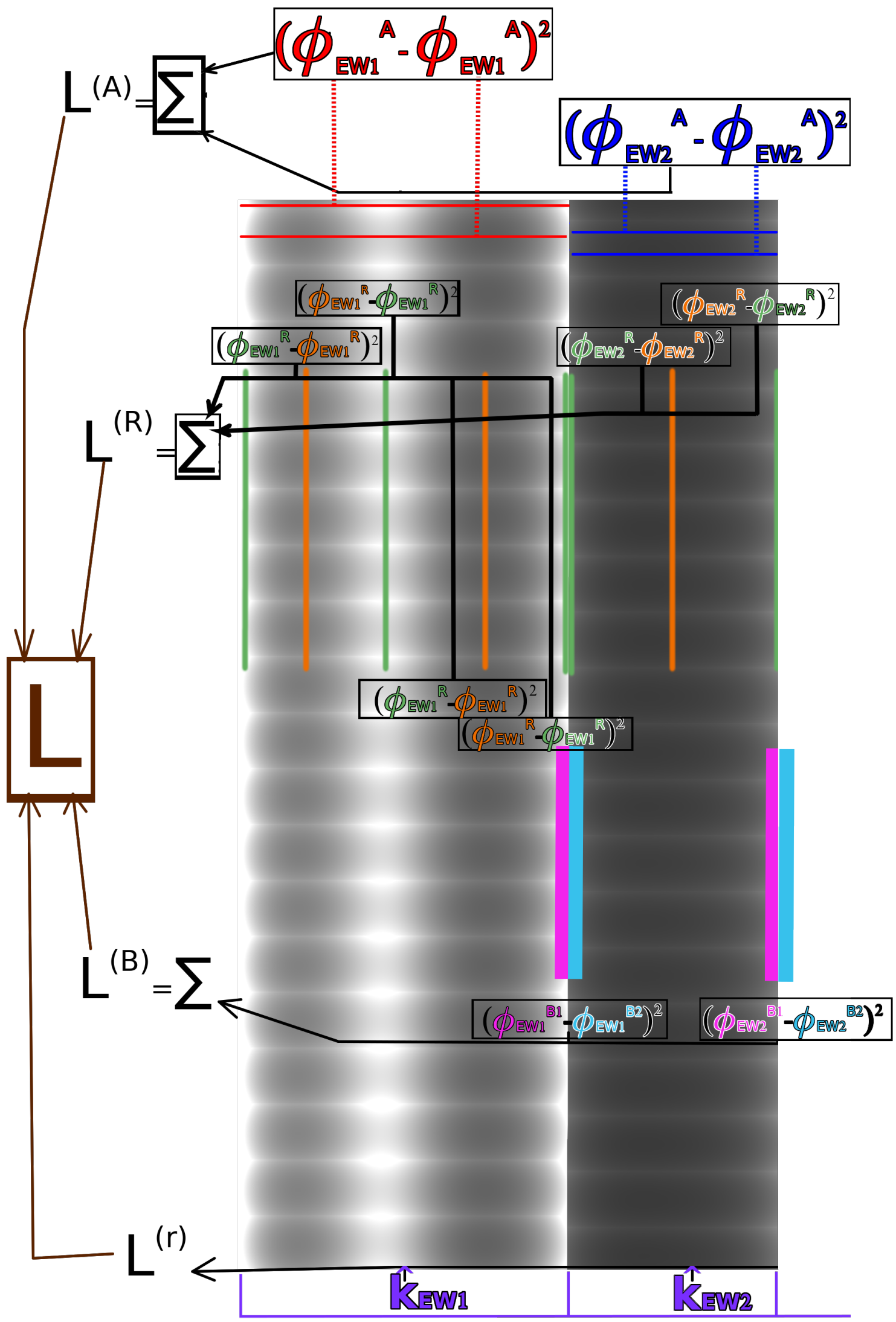

Figure 5: High level overview of the objective function for the first two subswaths, for simplicity. The noise field is overlaid with abridged annotations relating to the azimuth noise loss $\left(L^{A}\right)$, range noise loss $\left(L^{R}\right.$ and $\left.L^{B}\right)$, and regularization loss $\left(L^{r}\right)$. While the annotations are overlaid on the noise field to convey the indices of the points used in the terms, note that the loss is a function of the measured SAR image, the estimated noise field, and the scaling parameters. Best viewed in colour. 


\subsection{Objective: Azimuth}

A prominent attribute of the azimuth component of the noise is its periodic pattern. As explained previously, the pattern is caused by the U-shaped antenna pattern gain and the concatenation of bursts along the azimuth. Thus, the period of the azimuth noise is equivalent to the number of azimuth lines between the bursts, such that the troughs (local minima) of the azimuth noise correspond to centre of bursts (azimuth angle is 0) and are each one period apart. Also, since the gain is U-shaped, each peak (local maxima) of the azimuth noise is located half a period away from a trough. Therefore, we assert that if the amplitude of the noise is high, then the difference between the adjacent peaks and troughs will be high.

More generally, since the estimated noise within each burst is U-shaped and symmetric, we assume that the squared difference in the image between any pair of points along the azimuth will be proportional to the amplitude of the noise. Therefore, the pairs are selected as all possible points along the azimuth within each subswath paired with the points offset by half a period. Fig. 6a graphically shows this layout with respect to the azimuth.

Given the denoising model for subswath $a$ as $\hat{\phi}_{a}$, let $\hat{\phi}_{a}^{A}(i)$ be the average value of all pixels for azimuth line $i$ in $\hat{\phi}_{a}$. Then the azimuth component of the loss function is composed as

$$
L^{A}=\sum_{a \in \mathscr{A}} \sum_{i}^{N_{a z}(a)}\left[w_{a}^{A}(i)\left[\hat{\phi}_{a}^{A}(i)-\hat{\phi}_{a}^{A}(i+\rho(a))\right]\right]^{2},
$$

where $\rho(a)$ is the number of azimuth lines per half a burst period, computed as

$$
\rho(a)=\frac{N_{\text {burst }}(a)}{2 N_{a z}(a)}
$$

and $N_{\text {burst }}(a)$ is the number of bursts used to construct subswath $a$ and $N_{a z}(a)$ is the total number of azimuth lines in the subswath. Unfortunately, information containing $N_{\text {burst }}(a)$ is not explicitly included in the current IPF version (2.9). However, it can be derived as $N_{\text {burst }}(a)=N_{a p}(a)+1$, where $N_{a p}$ is the number of antennaPattern items for subswath $a$ within the annotation XML file. The term $w_{a}^{A}(i)$ is a weighting term introduced for subswath $a$ and azimuth line $i$ based on the realization that the change in intensity between pairs of lines separated by half a burst is influenced by the backscatter of the ground targets. If the two targets have fundamentally different backscattering properties, for example where one line is dominated by ice and the other is dominated by water, then their squared difference will not be representative of the scale of the noise field in the measurement. Given $x_{a}^{A}$ and $y_{a}^{A}$ as the average values on an azimuth line that are used in $\hat{\phi}_{a}^{A}$, the term $\frac{x_{a}^{A}(i)-x_{a}^{A}(i+\rho(a))}{y_{a}^{A}(i)-y_{a}^{A}(i+\rho(a))}$ is used to determine whether a sample's difference is dominated by the change in intensity from the backscattering of the targets. We determined an acceptable range as 0 to 2.5. The lower bound was zero because a negative ratio cannot be created from the additive noise, assuming the noise model is correct. A liberal upper bound was chosen as 2.5 based on experimentation. Thus, the weighting term is defined as

$$
w_{a}^{A}(i)=\left\{\begin{array}{rr}
1 & \text { if } 0<\frac{x_{a}^{A}(i)-x_{a}^{A}(i+\rho(a))}{y_{a}^{A}(i)-y_{a}^{A}(i+\rho(a))}<2.5 \\
0 & \text { otherwise }
\end{array}\right\}
$$

to remove the outlying pairs from the loss function.

As proven in the appendix, 60 can be represented using an inner product formulation

$$
L^{A}=\left[\mathbf{v}^{A}-\mathbf{C}^{A} \hat{\mathbf{k}}\right]^{T}\left[\mathbf{v}^{A}-\mathbf{C}^{A} \hat{\mathbf{k}}\right] .
$$

\subsection{Objective: Range}

As noted previously, the noise pattern in the range direction is unique for each subswath. Thus, two objective function terms, $L^{R}$ and $L^{B}$, are proposed to compensate the noise within the subswaths (intra-subswath) and between the subswaths (inter-subswath).

\subsubsection{Intra-subswath}

First we examine the intra-subswath loss term that describes the noise pattern within each subswath. Like the azimuth loss term, the intra-subswath loss term is constructed by taking the squared difference between peaks and troughs but within the range component. The range noise pattern is neither periodic nor symmetric; instead the true scale of the noise is indirectly measured from taking the squared difference between peaks and troughs 


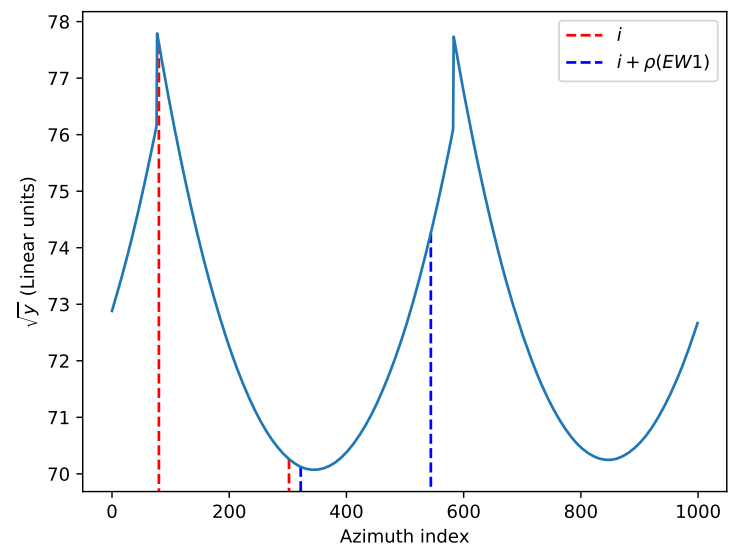

(a) Noise with respect to azimuth in EW1. The dashed lines show pairs of points that are offset by half a burst period that are used by the objective function.

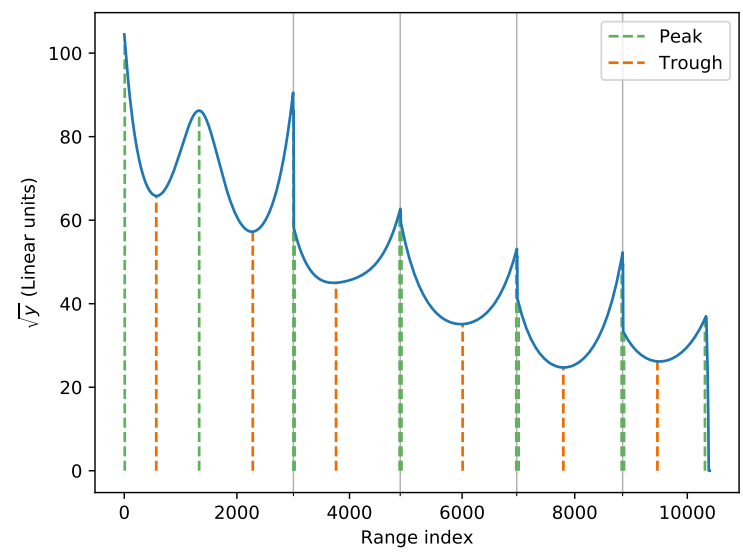

(b) Range noise with peaks and troughs over all five subswaths. The solid lines divide the subswaths, with the troughs and peaks marked by the dashed lines.

Figure 6: Peaks and troughs of the estimated noise with respect to azimuth and range.

exclusively. Fig. 6b shows the samples used with respect to the range direction. The samples used in the intrasubswath term are based on the rectangular subregions divided along the azimuth of a subswath that are dictated by the swathBoundList field in the noise calibration XML file, as depicted in Fig. 7 . The intra-subswath loss is based on the difference between adjacent peaks and troughs over each of the subregions. Let $p_{b}(z)$ and $t_{b}(z)$ be the $z^{\text {th }}$ peak and trough respectively within rectangular subregion $b$ in subswath $a$. Now let $\hat{\phi}_{b}^{R}(s)$ be the mean average of values predicted by the denoising model $\hat{\phi}$ within rectangular subregion $b$ in subswath $a$ that have a range index within $s-\epsilon$ to $s+\epsilon$, with a padding constant $\epsilon$ and $s$ being either $p_{b}(z)$ or $t_{b}(z)$. Since the range noise pattern is unimodal in EW2, EW3, EW4, and EW5 (loss term $M$ ), while multi-modal in EW1 (loss term $N$ ), the intra-subswath loss is composed as the sum of two terms

$$
L^{R}=M+N,
$$

where

$$
\begin{aligned}
M=\sum_{a \in \mathscr{M}} \sum_{b \in a}[ & {\left[w_{b}^{R}(1)\left[\hat{\phi}_{b}^{R}\left(p_{b}(1)\right)-\hat{\phi}_{b}^{R}\left(t_{b}(1)\right)\right]\right]^{2} } \\
& \left.+\left[w_{b}^{R}(2)\left[\hat{\phi}_{b}^{R}\left(t_{b}(1)\right)-\hat{\phi}_{b}^{R}\left(p_{b}(2)\right)\right]\right]^{2}\right] \\
& \text { such that } \mathscr{M}=\{\mathrm{EW} 2, \mathrm{EW} 3, \mathrm{EW} 4, \mathrm{EW} 5\}
\end{aligned}
$$

and

$$
\begin{aligned}
N=\sum_{b \in \mathrm{EW} 1}[ & {\left[w_{b}^{R}(1)\left[\hat{\phi}_{b}^{R}\left(p_{b}(1)\right)-\hat{\phi}_{b}^{R}\left(t_{b}(1)\right)\right]\right]^{2} } \\
& +\left[w_{b}^{R}(2)\left[\hat{\phi}_{b}^{R}\left(t_{b}(1)\right)-\hat{\phi}_{b}^{R}\left(p_{b}(2)\right)\right]\right]^{2} \\
& +\left[w_{b}^{R}(3)\left[\hat{\phi}_{b}^{R}\left(p_{b}(2)\right)-\hat{\phi}_{b}^{R}\left(t_{b}(2)\right)\right]\right]^{2} \\
& \left.+\left[w_{b}^{R}(4)\left[\hat{\phi}_{b}^{R}\left(t_{b}(2)\right)-\hat{\phi}_{b}^{R}\left(p_{b}(3)\right)\right]\right]^{2}\right]
\end{aligned}
$$

As in the azimuth noise component, weighting terms $w^{R}$ were introduced to account for instances where the difference is dominated by the difference in land targets (e.g. land vs ice). In this case the weighting term is defined as

$$
w_{b}^{R}(o)=\left\{\begin{array}{rr}
\mu & \text { if } 0<\frac{x_{b}^{R 1}-x_{b}^{R 2}}{y_{b}^{R 1}-y_{b}^{R 2}}<2.5 \\
0 & \text { otherwise }
\end{array}\right\},
$$

where $\hat{\phi}_{b}^{R}\left(p_{b}(o+q)\right)-\hat{\phi}_{b}^{R 2}\left(t_{b}(o)\right)=\left[x_{b}^{R 1}-k_{a} y_{b}^{R 1}\right]-\left[x^{R 2}-k_{a} y_{b}^{R 2}\right]$ with $o \in\{1,2\}$ and $q \in\{0,1\}$ such that they correspond with the difference terms in $\sqrt{11}$ and $\sqrt{12}$. The value $\mu$ balances the trade-off between $L^{A}$, which is summed over a larger number of terms. From experimentation we chose $\mu=1.79$.

Finally, the proposed error for the intra-subswath range noise can be rewritten as an inner product

$$
L^{R}=\left[\mathbf{v}^{R}-\mathbf{C}^{R} \hat{\mathbf{k}}\right]^{T}\left[\mathbf{v}^{R}-\mathbf{C}^{R} \hat{\mathbf{k}}\right],
$$


as shown in the appendix.

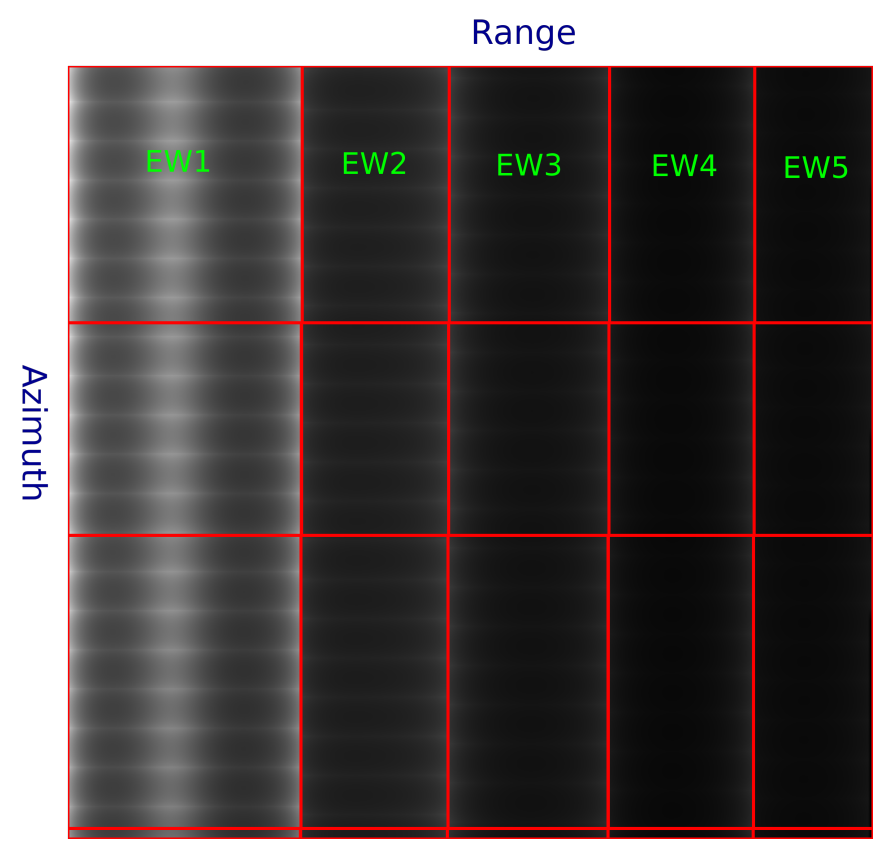

Figure 7: Layout of the rectangular subregions $b$ in red overlaid with the estimated noise pattern for reference.

\subsubsection{Inter-subswath}

As the radiation pattern changes when the antenna switches between subswaths, we must consider that this will cause discontinuities between adjacent subswaths. Intuitively, the range columns of adjacent subswaths are spatially correlated and should have similar values in an ideal denoised image. We introduce another loss term to represent the difference of intensity between the columns of adjacent subswaths to handle these considerations.

Let $\hat{\phi}_{b}^{B 1}$ be the average predicted value of the last $\epsilon$ range columns that are within subrectangle $b$ in subswath $a$ and let $\hat{\phi}_{b}^{B 2}$ be the average of the $\epsilon$ range columns after the last column in subrectangle $b$. Then the inter-subswath loss term is determined by

$$
\begin{aligned}
L^{B} & =\sum_{a \in \mathscr{B}} \sum_{b \in a}\left[\hat{\phi}_{b}^{B 1}-\hat{\phi}_{b}^{B 2}\right]^{2} \\
& =\left[\mathbf{v}^{B}-\mathbf{C}^{B} \hat{\mathbf{k}}\right]^{T}\left[\mathbf{v}^{B}-\mathbf{C}^{B} \hat{\mathbf{k}}\right] \\
& \text { such that } \mathscr{B}=\{\mathrm{EW} 1, \mathrm{EW} 2, \mathrm{EW} 3, \mathrm{EW} 4\}
\end{aligned}
$$

\subsection{Regularization}

Even with weight terms in the loss function, using each of the aforementioned loss terms can result in unstable estimates $\hat{\mathbf{k}}$. To resolve this, a prior assumption is made that each value of $\hat{\mathbf{k}}$ should be close to 1 . Thus a regularization term is introduced to penalize estimates of $\hat{\mathbf{k}}$ that diverge from 1

$$
\begin{aligned}
L^{r} & =\sum_{a}\left[\lambda_{a}\left[1-\hat{k}_{a}\right]\right]^{2} \\
& =\left[\mathbf{1}-\mathbf{C}^{r} \hat{\mathbf{k}}\right]^{T}\left[\mathbf{1}-\mathbf{C}^{r} \hat{\mathbf{k}}\right],
\end{aligned}
$$

where $\mathbf{C}^{r}$ is the diagonal matrix containing the hyper-parameter vector $\boldsymbol{\lambda}$. By applying the Golden section search algorithm in a block-wise manner, we found appropriate values of $\boldsymbol{\lambda}=[0.1,0.1,6.75124,2.78253,10]$, used for all images. Now that we have introduced the loss terms, we now detail the implementation for estimating the scaling parameters for each subswath.

\subsection{Implementation}

Recall that the standard denoising method proposed by ESA recommends denoising as

$$
\phi_{a}=x_{a}-y_{a}
$$


As it is apparent that this is not sufficient in some scenarios, the desired model scales the noise factor according to each subswath $a$ with $\hat{k}_{a}$

$$
\hat{\phi}_{a}=x_{a}-\hat{k}_{a} y_{a}
$$

Through matrix and vector concatenation the final loss term is defined as the sum of all the previously derived terms in a single inner product

$$
\begin{aligned}
L & =L^{A}+L^{R}+L^{B}+L^{r} \\
& =[\mathbf{v}-\mathbf{C} \hat{\mathbf{k}}]^{T}[\mathbf{v}-\mathbf{C} \hat{\mathbf{k}}]
\end{aligned}
$$

and the solution for $\operatorname{argmin} L$ is well known (Boyd and Vandenberghe, 2004, Fieguth, 2010) to be the least-squares solution

$\hat{\mathbf{k}}$

$$
\hat{\mathbf{k}}=\left[\mathbf{C}^{T} \mathbf{C}\right]^{-1} \mathbf{C}^{T} \mathbf{v}
$$

\section{Experiments}

To evaluate the effectiveness of our method, two experiments were considered. The first experiment is a simulation where a set of RADARSAT SAR images without significant additive noise patterns are selected to demonstrate the abilities of the proposed method in a setting where the characteristics of the noise are directly controlled. The second experiment considers denoising Sentinel-1 EW images in both HV and VH polarization and evaluates the effectiveness by examining sub-regions of open-water.

\subsection{Parameter re-estimation simulation on RADARSAT}

The simulation experiment can be summarized by the following: first RADARSAT images were selected, a scaled noise field was added to the images, scaling parameters were re-estimated on the noisy image, followed by denoising the noisy image with the model and comparing the result with original ground truth images. The RADARSAT images were selected to ensure that a ground truth reference image was available and independent of the additive noise patterns specific to Sentinel-1 EW. The goal of this experiment is to test if the proposed method can accurately estimate the optimal scaling parameters and verify the quality of the denoised image compared to a true original.

The set of images used for the experiments were 20 RADARSAT HV images taken in the Beaufort Sea during 2010. A template SAR noise field was selected from a sample Sentinel-1 scent ${ }^{2}$ To ensure comparability, the values of the RADARSAT scene prior to adding noise were re-scaled to have a comparable signal to noise ratio as the original Sentinel-1 scene after denoising with the proposed method. The RADARSAT scene was also spatially re-scaled with linear interpolation to have the same number of rows and columns as the Sentinel-1 scene.

For each image, 10 different noisy versions were constructed with scaling factors randomly selected from uniform distributions within the ranges $k_{\mathrm{EW} 1}:[1.2,1.6], k_{\mathrm{EW} 2}:[0.8,1.0], k_{\mathrm{EW} 3}:[0.92,1.02], k_{\mathrm{EW} 4}:[0.95,1.05], k_{\mathrm{EW} 5}:$ $[0.98,1.02]$, resulting in a total of 110 noisy images. These ranges for $\mathbf{k}$ were selected as they were representative of the range of estimated parameters encountered in the sample of true SAR images discussed in the following subsection. The noisy images were created by multiplying the noise field with the scaling parameters and adding it with the original image. Then, using $(20)$, the parameters were estimated, with the scaled subtraction labelled as the proposed denoising. As a baseline, we perform the aforementioned procedure using scaling estimates as $\overline{\mathbf{k}}=[1.4,0.925,0.985,1.0,1.0]$, which are the central values of the distributions. In addition, a visual comparison is shown in Fig. 8.

\footnotetext{
${ }^{2}$ The sample scene was from S1A_EW_GRDM_1SDH_20180902T164932_20180902T165032_023522_028FAA_5A8B
} 


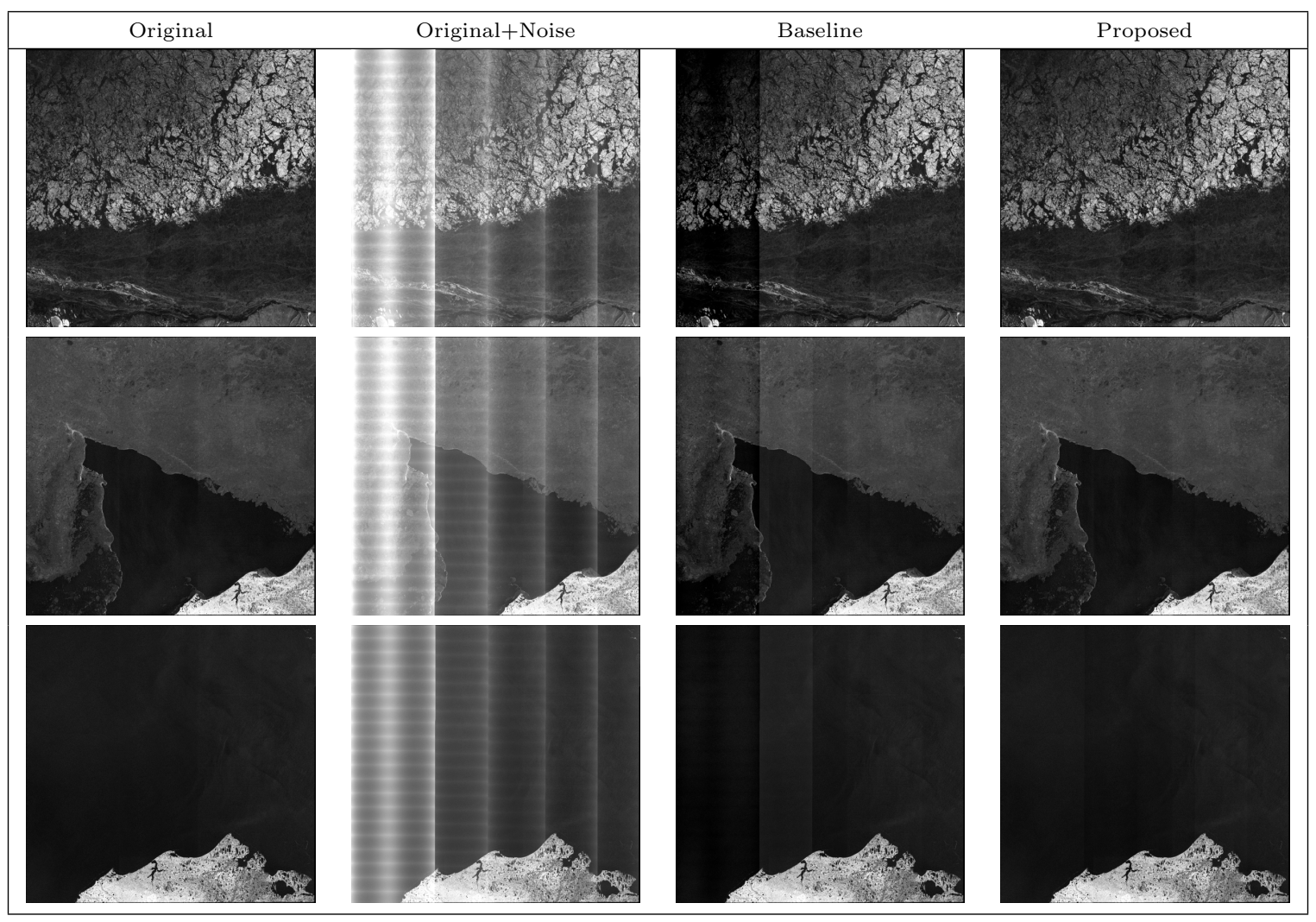

Figure 8: Comparison of images within the simulation experiment with $\hat{k}=[1.55,0.833,1.01,1.01,1.01]$. The proposed method is almost identical to the original image, while the image from the baseline method is scaled improperly between the simulated first and second subswaths.

Three metrics, the normalized root mean squared error (NRMSE), peak signal to noise ratio (PSNR), and structural similarity index (SSIM) (Wang et al. 2004), were computed with respect to the original image to determine the effectiveness of denoising. NRMSE computes the square root of the aggregate sum of pixel-wise squared differences, with a lower-bound of 0 indicating a perfect comparison. In contrast to root mean squared error, NRMSE is normalized by dividing the root sum of squares by the range of input values for each image, which helps ground the metric against a comparison that vary in scale (Alparone et al., 2015). We use a common variant of NRMSE as

$$
N R M S E(\mathbf{d}, \mathbf{l})=\frac{\sqrt{M S E(\mathbf{d}, \mathbf{l})}}{\max (\mathbf{l})-\min (\mathbf{l})}
$$

where $M S E(\mathbf{s}, \mathbf{l})$ is the mean squared error between prediction vector $\mathbf{d}$ and baseline vector $\mathbf{l}$. PSNR computes the logarithmic ratio of power of the original image to the power of the noise within the modified image. PSNR is commonly used in applications that result in distortion of the original image in denoising or compression applications (Gonzalez and Woods, 2017; Huynh-Thu and Ghanbari, 2008). Finally, SSIM is a metric bounded between 0 and 1 , with 1 indicating the two images are identical, and is commonly used for evaluating denoising and reconstruction methods by quantifying the similarity of structural features within an image (Wang et al. 2004). Each of these metrics make use of the available ground truth reference image. The summary of the metrics with respect to the denoising procedures over the distribution of images are shown in Table 1, with the metrics for the applying no denoising (noisy) included for context.

To compare the generated distributions between the proposed and baseline methods for each of the metrics, we employed a one-tailed paired t-tests using a critical value $\alpha=0.05$. We found our method had significantly higher SSIM and PSNR and lower NRMSE than the baseline method $(p<\alpha)$, indicating it is significantly better in terms of all three metrics. 
Table 1: Mean \pm standard deviation for the normalized root mean squared error (NRMSE), peak signal to noise ratio (PSNR), and structural similarity index (SSIM) over all the images with 10 iterations of sampling values for $k$. P-values from the one-tailed t-test comparing the proposed method to the other methods are also shown.

\begin{tabular}{|c||cc|cc|cc|}
\hline Method & NRMSE & p-value & PSNR & p-value & SSIM & p-value \\
\hline Noisy & $0.514 \pm 0.028$ & $\approx 0.0$ & $20.9 \pm 2.0$ & $\approx 0.0$ & $0.913 \pm 0.009$ & $\approx 0.0$ \\
Baseline $\mathbf{k}$ & $0.061 \pm 0.031$ & $1.5 \times 10^{-47}$ & $41.4 \pm 5.6$ & $3.8 \times 10^{-57}$ & $0.993 \pm 0.005$ & $9.8 \times 10^{-23}$ \\
Proposed & $\mathbf{0 . 0 1 7} \pm \mathbf{0 . 0 0 8}$ & N/A & $\mathbf{5 1 . 4} \pm \mathbf{3 . 8}$ & N $/ A$ & $\mathbf{0 . 9 9 7} \pm \mathbf{0 . 0 0 1}$ & N/A \\
\hline
\end{tabular}

\subsection{Denoising Sentinel-1 SAR}

For the second experiment, a set of 41 Sentinel-1 GRDM SAR images was selected to include 13 images from the Arctic Ocean, 6 from the Antarctic Ocean, 8 from the Atlantic Ocean, 6 from the Pacific Ocean, and 8 from the Indian Ocean. The images were selected from all five oceans to ensure our evaluation would not be biased towards a single environment. The images from the Arctic and Antarctic oceans have HV polarization while the images from the Atlantic, Pacific, and Indian oceans have VH polarization. The polarization of the images is determined by the Sentinel-1 observational scenario (CSC Mission Management Team, 2018). In the remainder of this section we provide both a visual and a quantitative comparison of the proposed and baseline denoising procedures.

Fig. 10 and Fig. 11 visually present a subset of co-polarized images from all five major ocean divisions. Three types of images are shown: the original image, the scene with ESA denoising, and the scene with a scaled noise field scaled and subtracted via the proposed method. For display purposes only, the images were linearly scaled from 0 to 255 for each scene based on the 2.5 to 97.5 percentile of the distribution of intensities for all versions of the scene in order to maximize the contrast in each image. Visually, the proposed method greatly reduces the noise characteristics from each of the images, although there are still some discontinuities in some of the images. This is not an error in the parameter estimation, but rather in the assumption that the noise field is linearly mis-scaled. More detail regarding this is provided in Section 5.

For the quantitative comparison of the experiment, we selected rectangular sub-regions in the image that spanned the entire range (all five subswaths) and were over open-water without major textural features. We make the assumption that the intensity over such sub-regions will not vary significantly with respect to range when the image is ideally denoised. A similar method of evaluation was also used in Park et al. (2018). Consequently, we assume that the mean intensity of these sub-regions should follow a linear relationship, with deviation from a linear relationship implying the presence of noise. Let $\xi(j)$ be the mean value along the azimuth on range index $j$ for a denoised image. Let $\hat{\xi}(j)$ be the linear fit for $\xi(j)$, which as previously mentioned, should be a reasonable estimate for $\xi(j)$. Fig. 9 shows an example of this process. The NRMSE between $\xi(j)$ and $\hat{\xi}(j)$ was then compared for the ESA denoising method and our proposed denoising method. The mean and standard deviation of NRMSE over all images are shown in Table 2. Using a one-tailed paired t-test, we found that our method had significantly lower NRMSE than the ESA method $\left(p=9.8 \times 10^{-11}<0.05\right)$. 

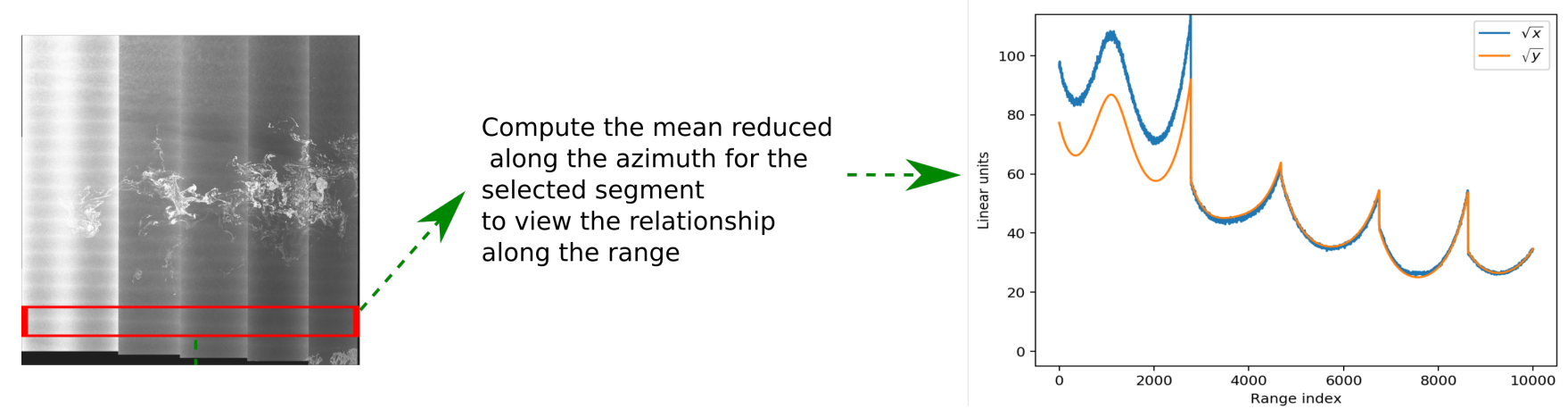

(a) Ocean value selection process
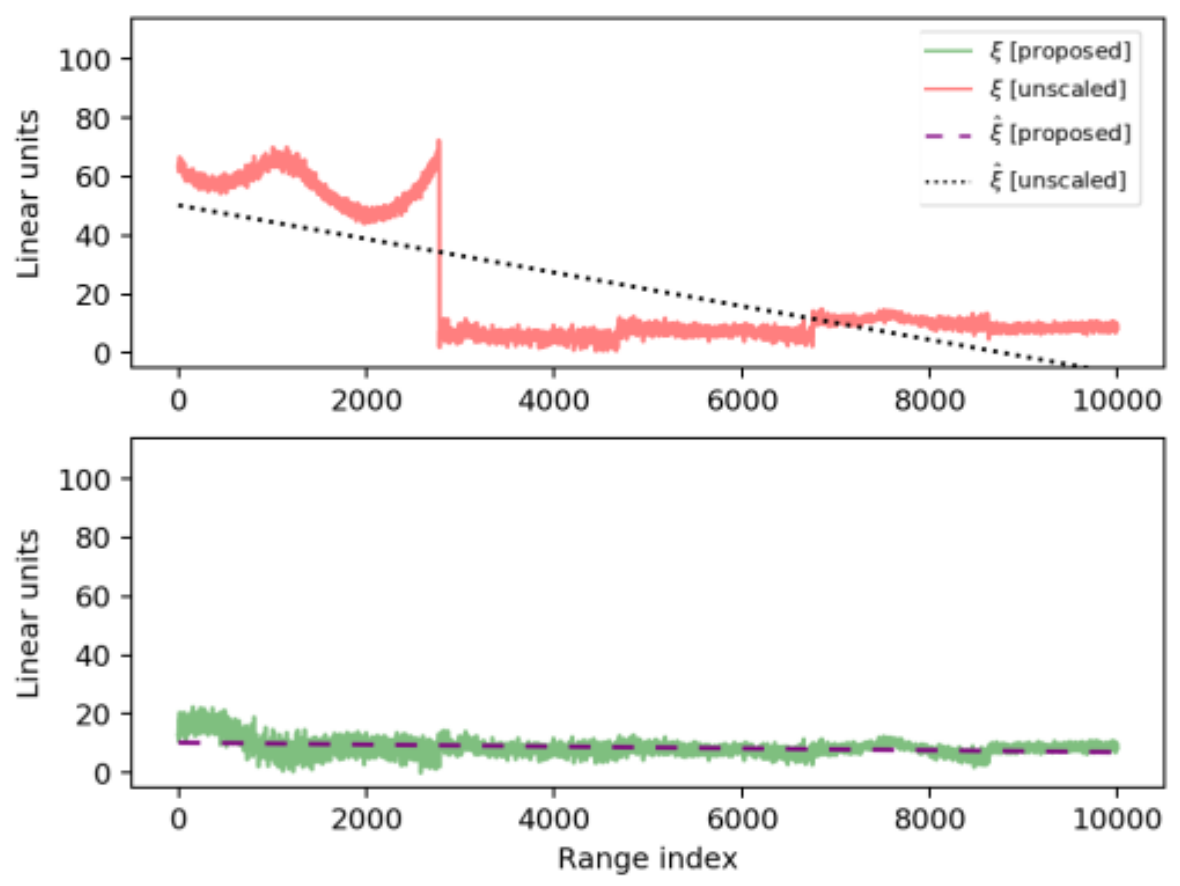

(b) Corresponding linear regression for the ESA method (top) and our method (bottom) using the mean values selected from Fig. 9a

Figure 9: Visualization of the quantitative experiment detailed in section 4.2

Table 2: Normalized root mean squared error for each image over selected ocean sub-images along with p-values generated from a one-tailed T-test comparing the proposed method to the others. The table is divided into comparing data from IPF 2.91 specific to sections 4.2 and 4.2 .1 and $3+$ that is specific to section 4.2 .2

\begin{tabular}{|c|cc||cc|}
\hline \multirow{2}{*}{ Method } & \multicolumn{2}{|c||}{ IPF 2.91} & \multicolumn{2}{c|}{ IPF $3+$} \\
\hline NSA & $1.85 \pm 0.941$ & $9.8 \times 10^{-11}$ & $0.685 \pm 0.278$ & 0.017 \\
Static $\bar{k}$ & $0.814 \pm 0.674$ & 0.0058 & N $/ \mathrm{A}$ & N/A \\
Proposed & $\mathbf{0 . 5 6 8} \pm \mathbf{0 . 2 7 4}$ & N/A & $\mathbf{0 . 5 9 2} \pm \mathbf{0 . 2 9 5}$ & N/A \\
\hline
\end{tabular}

\subsubsection{Static versus dynamic analysis}

While the overall effectiveness of the proposed method for denoising the SAR images has been demonstrated, there still remains the hypothesis that the ideal scaling parameters are different for each image. This hypothesis was tested by computing the aggregate mean values of $\hat{\mathbf{k}}$ with the proposed method for $\mathrm{HV}(\overline{\mathbf{k}}=[1.438,0.942,0.980,1.010,0.999])$ and VH $(\overline{\mathbf{k}}=[1.37,0.932,0.969,0.993,1.000])$ and comparing the effect of scaling the noise field with $\overline{\mathbf{k}}$ versus scaling 


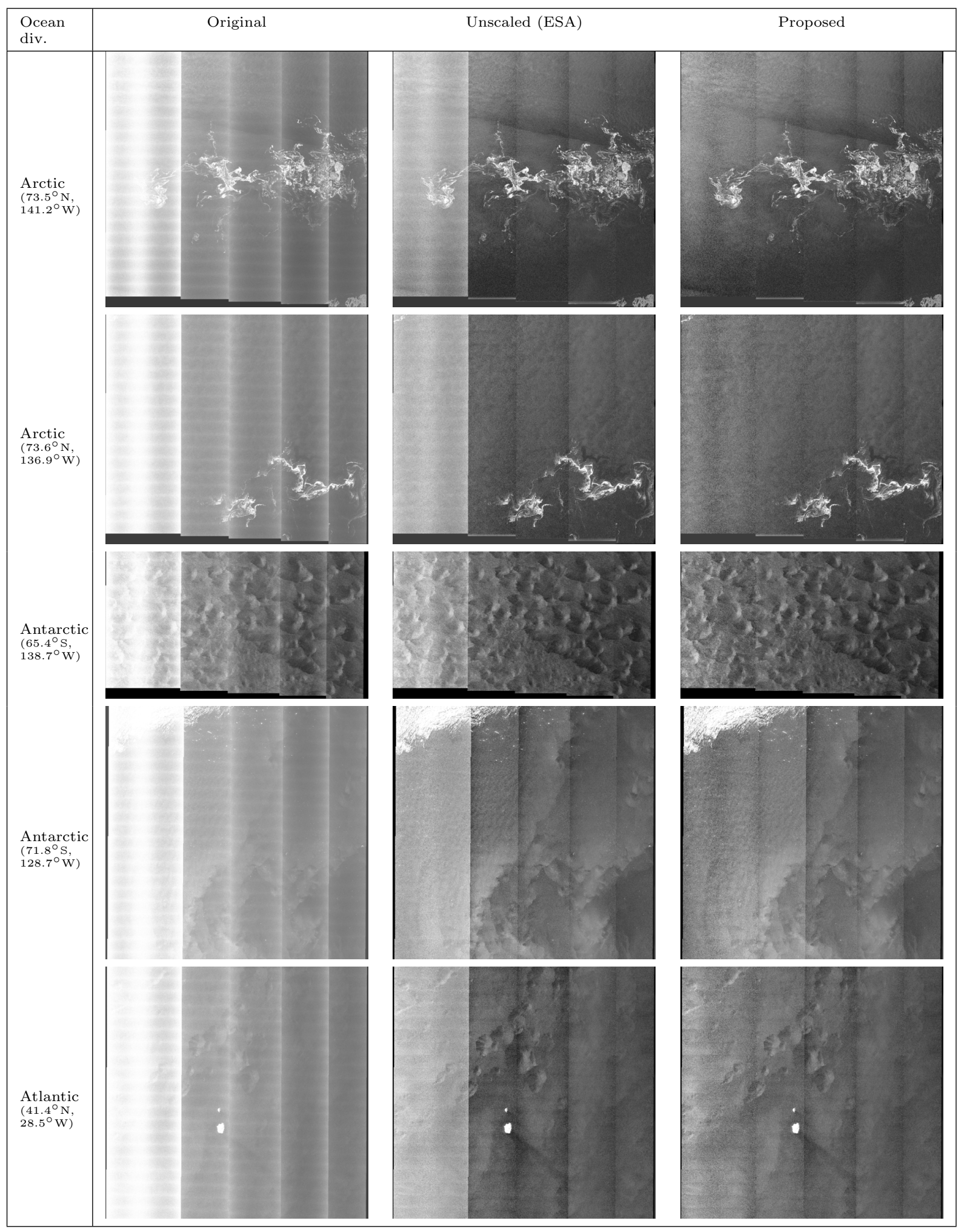

Figure 10: Part 1 gallery of SAR images showing the effect of the baseline and the proposed noise removal methods in reference to the original image. 


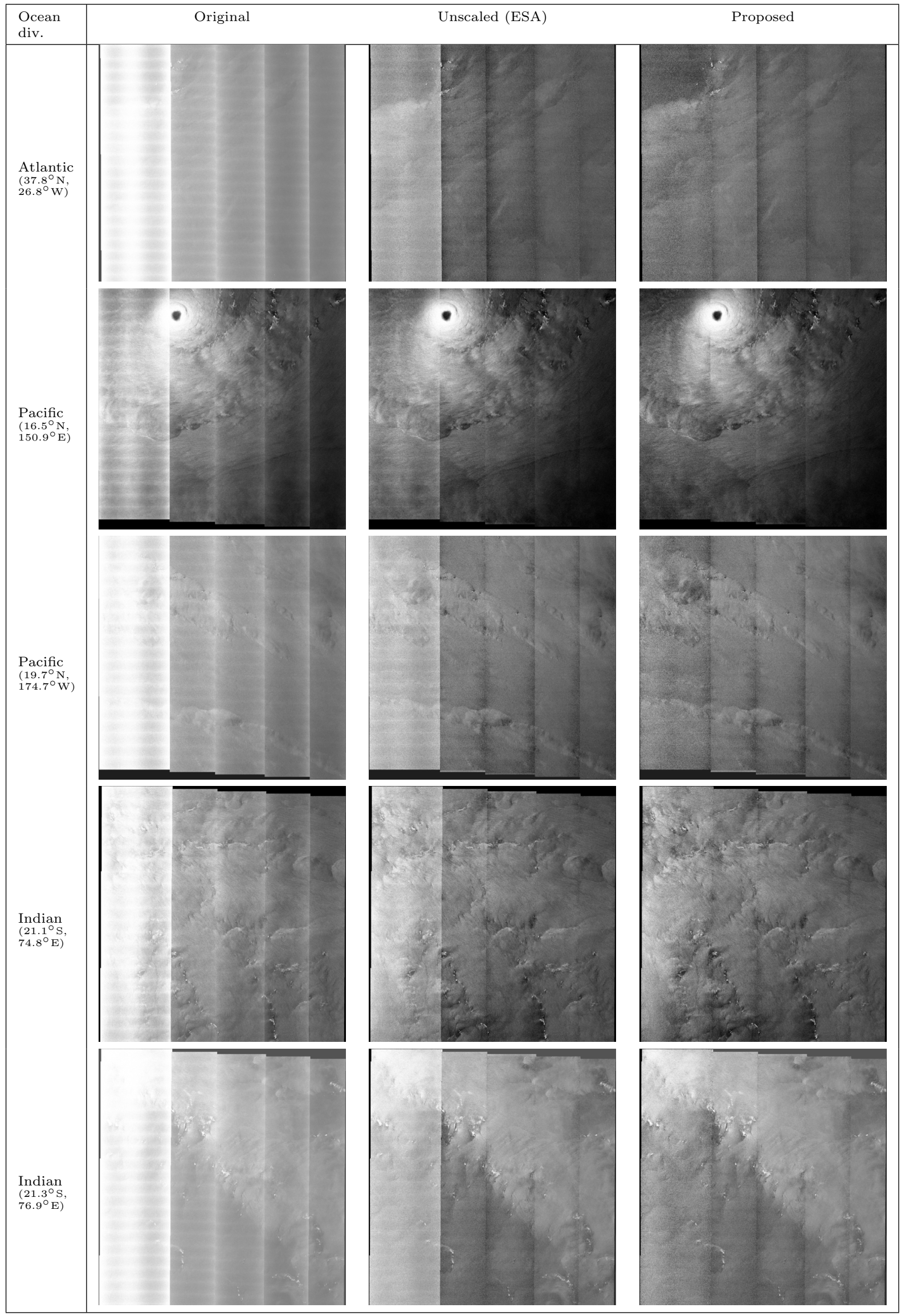

Figure 11: Part 2 gallery of SAR images showing the effect of the baseline and the proposed noise removal methods in reference to the original image. 
the noise field by dynamically estimating $\hat{\mathbf{k}}$ for each image. Fig. 12 shows an example of how using a static estimate $(\overline{\mathbf{k}})$ can provide sub-optimal denoising compared to using a dynamic estimate $(\hat{\mathbf{k}})$.

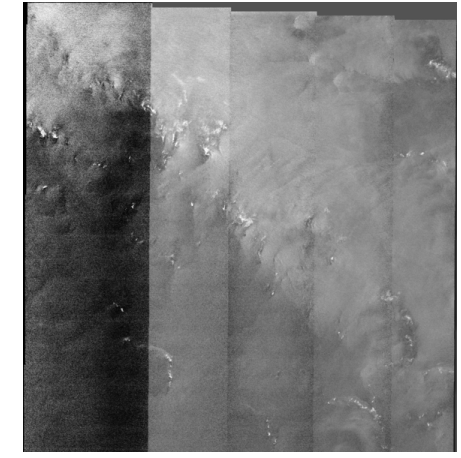

(a) Static scaling

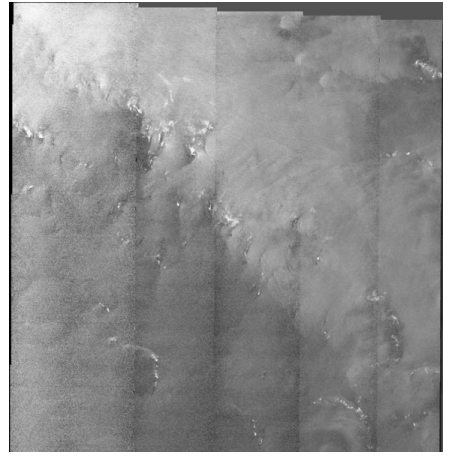

(b) Proposed dynamic scaling

Figure 12: An example of the importance of dynamically estimating scaling parameters for each image. In this case, the static scaling in this case overcompensates the true noise present in the image within the first swath.

To test the significance of this effect, the same quantitative experiment in section 4.2 , which compared the NRMSE of the linear regression with respect to the denoised measurement over ocean regions between two different methods, was applied. Namely, the mean estimates for $\overline{\mathbf{k}}$ were applied to scale the noise field as the static method versus scaling with $\hat{\mathbf{k}}$ using the proposed method. Overall, the proposed method had significantly lower NRMSE than the static $\left(p=5.8 \times 10^{-3}<0.05\right)$ from a one-tailed t-test, thus supporting our hypothesis that the ideal scaling factors are scene independent. Once again, these results are summarized in Table 2.

\subsubsection{IPF 3+}

With the changes from IPF version $3+$, the generation of noise fields changed significantly. A new normalization scheme was added to help account for situations where the signal to noise ratio is low (Piantanida, 2019). To evaluate the effect of the proposed method on these new noise fields, we collected 13, 12, 8, 14, and 9 images from the Arctic, Antarctic, Atlantic, Pacific, and Indian oceans with sensing dates after July 2019. We repeated the quantitative experiment with this data, while skipping the static comparison for brevity. Table 2 indicate that the proposed method (NRMSE $=0.592$ ) had significantly lower NRMSE than the ESA method (NRMSE $=0.685)$, with $p=0.017<0.05$. As shown in Figure 13 , the improvement between the proposed method and the ESA method is milder than with IPF 2.91.
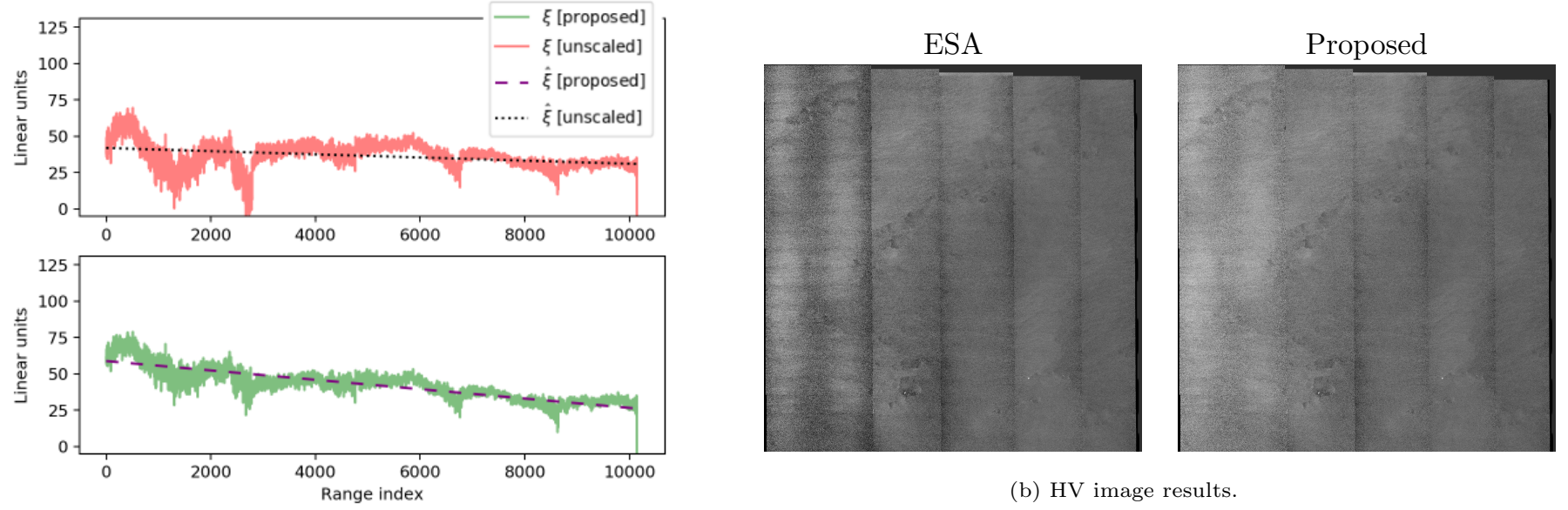

(b) HV image results.

(a) Range plot and linear regression for ESA method (top) and the proposed method(bottom)

Figure 13: Brief comparison with IPF version 3+ 


\section{Discussion}

As mentioned in the introduction, the merit of the proposed method is based on achieving four objectives. The first three objectives are directly tied to the results of the two experiments, while the last objective is achieved from the formulation of the method itself.

The simulation experiment is based on the ideal scenario where our assumptions of actual additive noise are true; in other words, the noise field is linearly mis-scaled between subswaths and the scaling is variable among different scenes. Under these circumstances, the proposed method provides an almost perfect denoising of the selection of Sentinel-2 scenes over coastal regions. In this scenario, the proposed method produced significantly better denoising than the scaling from using the static expected scaling parameters. This indicates that the proposed method can effectively adapt to the dynamic-scaling of the noise field in an ideal environment.

While the simulation experiment was a good demonstration that the proposed method works well in a controlled setting where the ground truth is known, obviously the characteristics of SAR and optical images are inherently different and the experiment on SAR images is required even if a ground truth reference is not known. Overall, the proposed method made dramatic improvements on the visual quality of the SAR images. The method was able to estimate parameters in a wide variety of backgrounds including those with heterogeneous textures, such as sea-ice or cyclones. Compared to subtracting an unscaled noise field, the proposed method suppresses the characteristics of the noise field to a much greater degree. Analytically, the evidence that the proposed method produces significantly lower NRMSE over the range of ocean regions concurs to the effectiveness of the proposed noise removal and the accomplishment of the first two objectives.

The experiment on the SAR images also revealed the impact of dynamically estimating scaling parameters. By using the mean parameters generated by the proposed method as the static scaling parameters, there are instances where the static parameters are unsuitable for the subswaths. This effect was significant in terms of NRMSE over range of ocean regions, indicating that there is a clear benefit for dynamic estimation.

Also of note is that the proposed method requires little computational overhead relative to the size of the noise field and image. The computational complexity of constructing the linear system is of the same order as a reduction along the azimuth, while solving the $5 \times 5$ linear system of least-squares is trivial. The small size of the system is also beneficial because a closed form solution can be used and no expensive iterative algorithms like the conjugate gradient method, gradient descent, or grid search are required to estimate scaling parameters. The low overhead is another advantage of the dynamic parameter estimation aspect of the proposed method because the parameters can be quickly estimated for each image and, unlike a static estimation approach, requires no preparation in the sense of collecting a training set.

The introduction of IPF $3+$ greatly improved the quality of the noise field. In section 4.2 .2 , it was demonstrated that the proposed method still provided some modest improvement, but the visual differences can be subtle. However, archived Sentinel-1 data is not updated with the newest IPF and are still susceptible to the mis-scale from IPF 2.91 and below. Therefore, our method provides a way for improving quality that can be applied to both older and current Sentinel-1 data.

Despite the aforementioned success, some issues still exist in the SAR-images. As mentioned in Section 4.2. some images have discontinuities between adjacent subswaths even after denoising. These issues do not seem to be from an incorrect estimation of scaling parameters. Rather, they seem to be caused from the shape of the ESA noise field not always fitting to the actual shape of noise imposed on the image, particularly in the range direction. An extreme example of this is shown in Fig. 14a, where the left and right extremities of subswaths EW2, EW3, EW4, EW5 have a higher relative compensation than the centre of the swath. Another example in Fig. 14b shows a different style of misfit, where the left extremities of the subswaths have higher relative compensation compared to the right extremity. This shows that for some images, the provided noise fields are calibrated incorrectly in a more complex manner than linear scale. Consequently, this misfit accounts for errors in the noise removal that cannot be compensated by scaling individual subswaths alone. Correcting this will require correcting the shape of the noise curves either through enhanced calibration or by creating a more flexible empirical model to account for the shape of the curves. This is consequently an area of study for future work. While it would be interesting to adapt these methods to co-polarized images or images from Sentinel-1 modes such as IW, we suspect that the higher signal-to-noise ratios from these methods would make application of our methods to these types of images less necessary. 


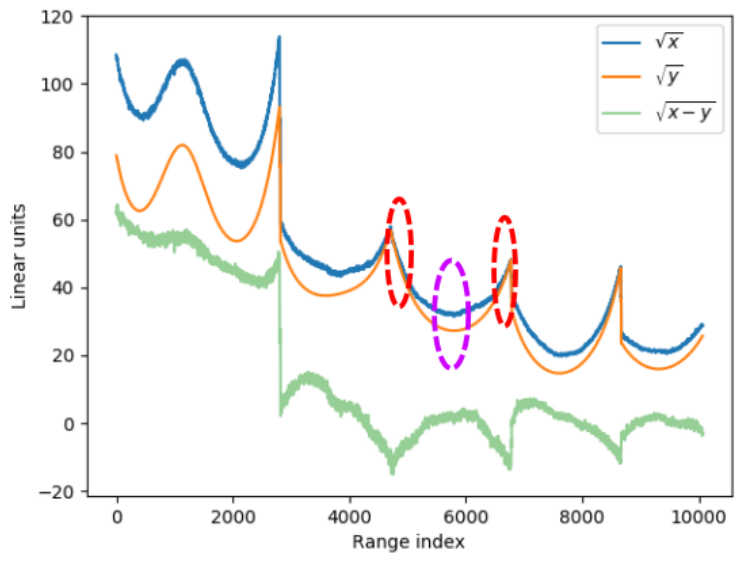

(a) ID: 20180930T081301_20180930T081401_023925_029CB0

The relative difference between the measurements and the noise is greater in the centre of subswaths (purple) than the right and left extremities (red) within the same subswath.

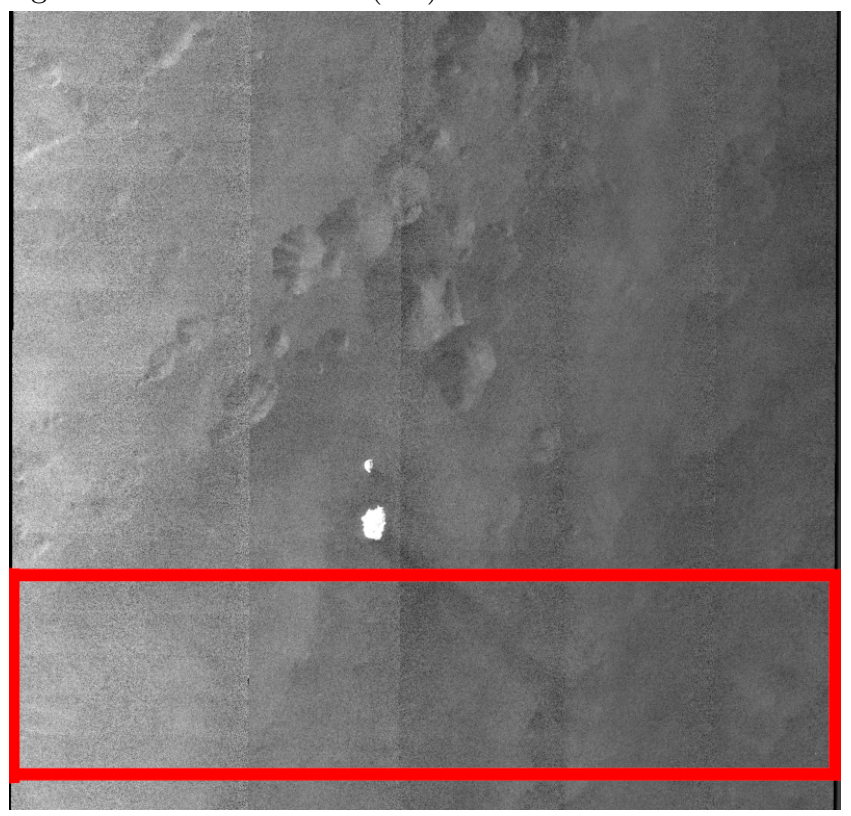

(c) ID: 20180930T081301_20180930T081401_023925_029CB0: Input image with evaluation range highlighted in red.

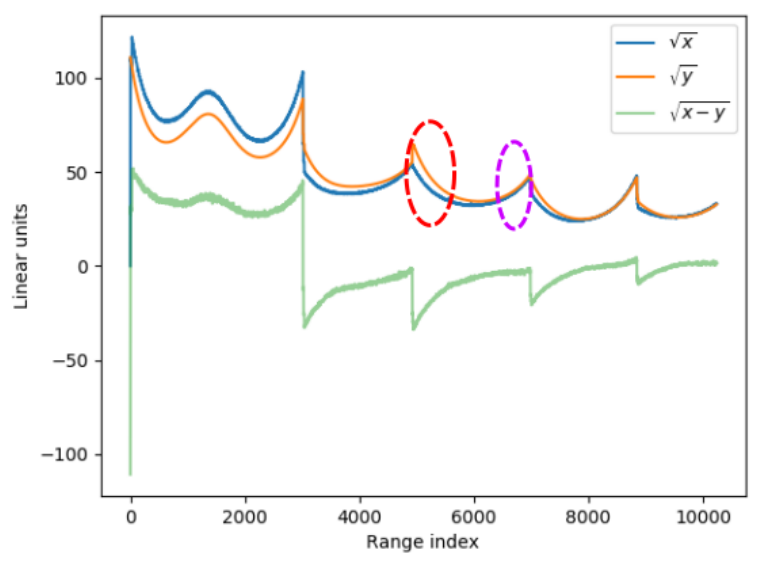

(b) ID: 20190322T212224_20190322T212324_015472_01CFB5: The relative difference between the noise and signal is different for the right (red) and left extremities (purple) within the same subswath.

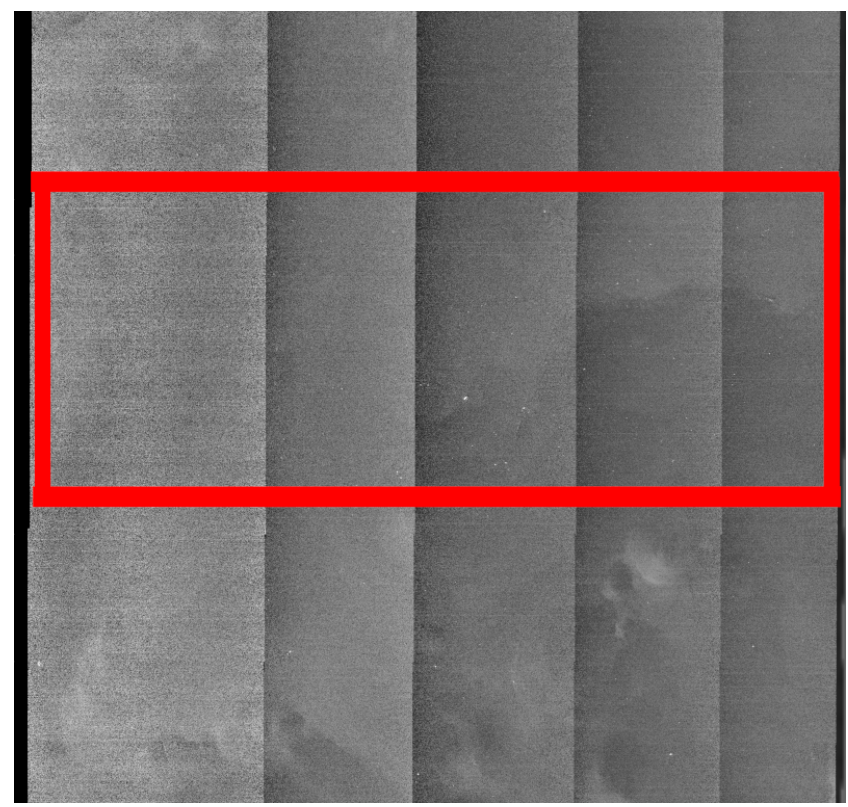

(d) ID: 20190322T212224_20190322T212324_015472_01CFB5: Input image with evaluation range highlighted in red.

Figure 14: Mean signal and noise with respect to range over an ocean sub-region. In both examples the noise is not fit properly for subswaths EW2, EW3, EW4, and EW5.

\section{Conclusion}

We created a quadratic objective function to model the characteristics of the estimated noise field in TOPSAR cross-polarized images. Our method uses this objective function to estimate scaling parameters for each of the subswaths in a scene. The method compared favourably to unscaled noise removal in both a simulation experiment on non-SAR images on an experiment selection of Sentinel-1 cross polarized TOPSAR images over major oceandivisions around the world. These experiments showed that the algorithm works on a variety of backgrounds, has no requirements of acquiring a training data set, provides dynamic scaling parameter estimation for each image, and has low computational requirements. These merits are beneficial for any practitioner who uses cross-polarized Sentinel-1 EW images, as the algorithm can be conveniently applied with little preparation required. 


\section{Conflict of Interest}

Declarations of Interest: None

\section{Acknowledgments}

We acknowledge the funding support of the Natural Sciences and Engineering Research Council of Canada (NSERC) (RGPIN-2017-04869, DGDND-2017-00078, RGPAS-2017-50794, RGPIN-2019-06744) and from the University of Waterloo.

Thanks are also extended to Prof. K. Andrea Scott, Mohsen Ghanbari, and Mingzhe Jiang for their feedback on the manuscript.

\section{Appendix A. Inner Product Formulations}

Claim. $L^{\sim} \in\left\{L^{A}, L^{B}, L^{R}\right\}$ can be represented with an arbitrary inner product formulation $\left[\mathbf{v}^{\sim}-\mathbf{C}^{\sim} \hat{\mathbf{k}}\right]^{T}\left[\mathbf{v}^{\sim}-\mathbf{C}^{\sim} \hat{\mathbf{k}}\right]$, using some vector $\mathbf{v}^{\sim}$ and matrix $\mathbf{C}^{\sim}$.

Proof. First, discard the connotations associated with the variables $i, j$, and $n$ in the main text. Without loss of generality, let $L^{\sim}=\sum_{i=1}^{N}\left[w(i)\left[\hat{\phi}_{a(2 i-1)}^{2 i-1}-\hat{\phi}_{a(2 i)}^{2 i}\right]\right]^{2}$ given the linear denoising model $\hat{\phi}_{a(j)}^{j}=x_{a(j)}^{j}-\hat{k}_{a(j)} y_{a(j)}^{j}$, with $x_{a(j)}^{j}$ representing a selection of the measurement, $y_{a(j)}^{j}$ representing a selection of the estimated noise, and a subswath $a(j) \in \mathscr{A}$. Then for any $i$,

$$
\begin{aligned}
w(i)\left[\hat{\phi}_{a(2 i-1)}^{2 i-1}-\hat{\phi}_{a(2 i)}^{2 i}\right] & =w(i)\left[x_{a(2 i-1)}^{2 i-1}-\hat{k}_{a(2 i-1)} y_{a(2 i-1)}^{2 i-1}\right]-\left[y_{a(2 i)}^{2 i}-\hat{k}_{a(2 i)} y_{a(2 i)}^{2 i}\right] \\
& =w(i)\left[x_{a(2 i-1)}^{2 i-1}-x_{a(2 i)}^{2 i}-\left[\hat{k}_{a(2 i-1)} y_{a(2 i-1)}^{2 i-1}-\hat{k}_{a(2 i)} y_{a(2 i)}^{2 i}\right]\right]
\end{aligned}
$$

We can encode A.1 for all $i$ into a vector $\mathbf{v}^{\sim}-\mathbf{C}^{\sim} \hat{\mathbf{k}}$ where $\mathbf{v}^{\sim}$ contains the terms with $x$ and $\mathbf{C}^{\sim}$ contains the terms with $y$. More precisely,

$$
\mathbf{v}^{\sim} \in \mathbb{R}^{N}=\left|\begin{array}{c}
w(1)\left[x_{a(1)}^{1}-x_{a(2)}^{2}\right] \\
\vdots \\
w(i)\left[x_{a(2 i-1)}^{2 i-1}-x_{a(2 i)}^{2 i}\right] \\
\vdots \\
w(N)\left[x_{a(2 N-1)}^{2 N-1}-x_{a(2 N)}^{2 N}\right]
\end{array}\right|
$$

and

$$
\mathbf{C}^{\sim}(i, n)=\left\{\begin{array}{lr}
w(i)\left[y_{a(2 i-1)}^{2 i-1}-y_{a(2 i)}^{2 i}\right] & \text { if } a(2 i-1)=a(2 i)=n \\
w(i) y_{a(2 i-1)}^{2 i-1} & \text { if } a(2 i-1)=n \text { and } a(2 i) \neq n \\
-w(i) y_{a(2 i)}^{2 i} & \text { if } a(2 i)=n \text { and } a(2 i-1) \neq n \\
0 & \text { otherwise }
\end{array}\right\},
$$

with the index $n$ representing the different subswaths $\in \mathscr{A}$ and $\mathbf{C}^{\sim} \in \mathbb{R}^{N \times 5}$. Then by the rules of matrix multiplication

$$
\sum_{i=1}^{N}\left[w(i)\left[\hat{\phi}_{a(2 i-1)}^{2 i-1}-\hat{\phi}_{a(2 i)}^{2 i}\right]\right]^{2}=\left[\mathbf{v}^{\sim}-\mathbf{C}^{\sim} \hat{\mathbf{k}}\right]^{T}\left[\mathbf{v}^{\sim}-\mathbf{C}^{\sim} \hat{\mathbf{k}}\right]
$$

\section{References}

Agrawal, N., Venugopalan, K., 2011. Speckle reduction in remote sensing images, in: 2011 International Conference on Emerging Trends in Networks and Computer Communications (ETNCC), pp. 195-199. doi $10.1109 /$ ETNCC.2011.5958515.

Alparone, L., Aiazzi, B., Baronti, S., Garzelli, A., 2015. Remote Sensing Image Fusion. CRC Press, Inc., Boca Raton, FL, USA. 
Bailey, G.B., 2014. Land Surface Topography. Springer New York, New York, NY. pp. 320-325. URL: https://doi.org/10.1007/978-0-387-36699-9_80, doi:10.1007/978-0-387-36699-9_80.

Boyd, S., Vandenberghe, L., 2004. Convex Optimization. Cambridge University Press. pp. 1-15.

CSC Mission Management Team, 2018. Sentinel High Level Operations Plan. Technical Report 2(2). Reference: COPE-S1OP-EOPG-PL-15-0020.

Elachi, C., Bicknell, T., Jordan, R.L., Chialin Wu, 1982. Spaceborne synthetic-aperture imaging radars: Applications, techniques, and technology. Proceedings of the IEEE 70, 1174-1209. doi:10.1109/PROC.1982.12448.

Fieguth, P., 2010. Statistical Image Processing and Multidimensional Modeling. Springer Science \& Business Media. pp. 13-51.

Gagnon, L., Jouan, A., 1997. Speckle filtering of SAR images: A comparative study between complex-wavelet-based and standard filters, in: Wavelet Applications in Signal and Image Processing V, International Society for Optics and Photonics. pp. 80-91.

Gonzalez, R.C., Woods, R.E., 2017. Digital Image Processing. 4 ed.. Pearson Education, Inc. pp. 595-687.

Hong, D.B., Yang, C.S., 2018. Automatic discrimination approach of sea ice in the Arctic Ocean using Sentinel-1 extra wide swath dual-polarized SAR data. International Journal of Remote Sensing 39, 4469-4483. URL: https://doi.org/10.1080/01431161.2017.1415486, doi:10.1080/01431161.2017.1415486, arXiv:https://doi.org/10.1080/01431161.2017.1415486.

Huynh-Thu, Q., Ghanbari, M., 2008. Scope of validity of PSNR in image/video quality assessment. Electronics Letters $44,1-2$.

Iqbal, M., Chen, J., Yang, W., Wang, P., Sun, B., 2012. Kalman filter for removal of scalloping and inter-scan banding in ScanSAR images. Progress In Electromagnetics Research 132, 443-461.

Karvonen, J., 2017. Baltic Sea ice concentration estimation using Sentinel-1 SAR and AMSR2 microwave radiometer data. IEEE Transactions on Geoscience and Remote Sensing 55, 2871-2883. doi $10.1109 /$ TGRS. 2017.2655567.

Kasischke, E.S., Melack, J.M., Dobson, M.C., 1997. The use of imaging radars for ecological applications-a review. Remote Sensing of Environment 59, 141 - 156. URL: http://www.sciencedirect.com/science/article/pii/S0034425796001484 doi:https://doi.org/10.1016/S0034-4257(96)00148-4, spaceborne Imaging Radar Mission.

Li, B., Liu, B., Guo, W., Zhang, Z., Yu, W., 2018. Ship size extraction for Sentinel-1 images based on dual-polarization fusion and nonlinear regression: Push error under one pixel. IEEE Transactions on Geoscience and Remote Sensing 56, 4887-4905. doi:10.1109/TGRS.2018.2841882.

Maity, A., Pattanaik, A., Sagnika, S., Pani, S., 2015. A comparative study on approaches to speckle noise reduction in images, in: 2015 International Conference on Computational Intelligence and Networks, pp. 148-155. doi $10.1109 /$ CINE.2015.36

Marhaba, B., Zribi, M., 2018. Reduction of speckle noise in SAR images using hybrid combination of bootstrap filtering and DWT, in: 2018 International Conference on Computer and Applications (ICCA), pp. 377-382. doi:10.1109/COMAPP. 2018.8460380.

Park, J.W., Korosov, A.A., Babiker, M., Sandven, S., Won, J.S., 2018. Efficient thermal noise removal for Sentinel-1 TOPSAR cross-polarization channel. IEEE Transactions on Geoscience and Remote Sensing 56, $1555-1565$.

Parrilli, S., Poderico, M., Angelino, C.V., Verdoliva, L., 2011. A nonlocal SAR image denoising algorithm based on LLMMSE wavelet shrinkage. IEEE Transactions on Geoscience and Remote Sensing 50, 606-616.

Pelich, R., Longépé, N., Mercier, G., Hajduch, G., Garello, R., 2015. Performance evaluation of Sentinel-1 data in SAR ship detection, in: 2015 IEEE International Geoscience and Remote Sensing Symposium (IGARSS), pp. 2103-2106. doi:10.1109/IGARSS.2015.7326217. 
Pepe, A., Calò, F., 2017. A review of interferometric synthetic aperture RADAR (InSAR approaches for the retrieval of Earth's surface displacements. Applied Sciences 7, 1264.

Piantanida, R., 2017a. Sentinel-1 Level 1 Detailed Algorithm Definition. Technical Report 2/1. European Space Agency. Reference: MPC-0307.

Piantanida, R., 2017b. Thermal Denoising of Products Generated by the S-1 IPF. Technical Report 1.1. European Space Agency. URL: https://sentinel.esa.int/documents/247904/2142675/ Thermal-Denoising-of-Products-Generated-by-Sentinel-1-IPF. Reference: MPC-0392.

Piantanida, R., 2019. Sentinel-1 Level 1 Detailed Algorithm Definition. Technical Report 2/2. European Space Agency. URL: https://sentinels.copernicus.eu/documents/247904/1877131/ Sentinel-1-Level-1-Detailed-Algorithm-Definition. Reference: MPC-0307.

Qiu, F., Berglund, J., Jensen, J.R., Thakkar, P., Ren, D., 2004. Speckle noise reduction in SAR imagery using a local adaptive median filter. GIScience \& Remote Sensing 41, 244-266.

Santoso, A.W., Pebrianti, D., Bayuaji, L., Zain, J.M., 2015. Performance of various speckle reduction filters on synthetic aperture radar image, in: 2015 4th International Conference on Software Engineering and Computer Systems (ICSECS), IEEE. pp. 11-14.

Tan, W., Li, J., Xu, L., Chapman, M.A., 2018. Semiautomated segmentation of Sentinel-1 SAR imagery for mapping sea ice in Labrador Coast. IEEE Journal of Selected Topics in Applied Earth Observations and Remote Sensing 11, 1419-1432. doi 10.1109/JSTARS.2018.2806640.

Topouzelis, K., 2008. Oil spill detection by SAR images: Dark formation detection, feature extraction and classification algorithms. Sensors 8, 6642-6659.

Visser, H., 2012. Antenna Theory and Applications. John Wiley \& Sons Ltd. pp. 189-227. doi $10.1002 / 9781119944751$

Wang, L., Scott, K.A., Clausi, D.A., Xu, Y., 2017. Ice concentration estimation in the Gulf of St. Lawrence using fully convolutional neural network, in: 2017 IEEE International Geoscience and Remote Sensing Symposium (IGARSS), pp. 4991-4994. doi 10.1109/IGARSS.2017.8128124.

Wang, Z., Bovik, A.C., Sheikh, H.R., Simoncelli, E.P., et al., 2004. Image Quality Assessment: From Error Visibility to Structural Similarity. IEEE Transactions on Image Processing 13, 600-612.

Wei, J., Chen, H., 2007. Effect of roll angle error on spaceborne ScanSAR radiometric calibration in range, in: 2007 International Symposium on Microwave, Antenna, Propagation and EMC Technologies for Wireless Communications, IEEE. pp. 1471-1475. doi 10.1109/MAPE.2007.4393558.

Xu, L., Li, J., 2015. Mapping Sea Ice from Satellite SAR Imagery. Springer Netherlands, Dordrecht. pp. 113-135. URL: https://doi.org/10.1007/978-94-017-9813-6_6, doi:10.1007/978-94-017-9813-6_6.

Xu, L., Shafiee, M.J., Wong, A., Clausi, D.A., 2016. Fully connected continuous conditional random field with stochastic cliques for dark-spot detection in SAR imagery. IEEE Journal of Selected Topics in Applied Earth Observations and Remote Sensing 9, 2882-2890. doi:10.1109/JSTARS.2016.2531985.

Yu, Q., Clausi, D.A., 2008. IRGS: Image segmentation using edge penalties and region growing. IEEE Trans. Pattern Anal. Mach. Intell. 30, 2126-2139. URL: http://dx.doi.org/10.1109/TPAMI.2008.15, doi $10.1109 /$ TPAMI.2008.15

Zan, F.D., Guarnieri, A.M., 2006. TOPSAR: Terrain observation by progressive scans. IEEE Transactions on Geoscience and Remote Sensing 44, 2352-2360. 